\title{
Extra U(1), effective operators, anomalies and dark matter
}

\author{
Emilian Dudas, ${ }^{a}$ Lucien Heurtier, ${ }^{a}$ Yann Mambrini ${ }^{b}$ and Bryan Zaldivar ${ }^{c}$ \\ ${ }^{a}$ CPhT, Ecole Polytechnique, \\ 91128 Palaiseau Cedex, France \\ ${ }^{b}$ Laboratoire de Physique Théorique, \\ Université Paris-Sud, F-91405 Orsay, France \\ ${ }^{c}$ Instituto de Fisica Teorica, IFT-UAM/CSIC, \\ 28049 Madrid, Spain \\ E-mail: Emilian.Dudas@cpht.polytechnique.fr, \\ heurtier@cpht.polytechnique.fr, yann.mambrini@th.u-psud.fr , \\ b.zaldivar.m@csic.es
}

ABSTRACT: A general analysis is performed on the dimension-six operators mixing an almost hidden $Z^{\prime}$ to the Standard Model (SM), when the $Z^{\prime}$ communicates with the SM via heavy mediators. These are fermions charged under both $Z^{\prime}$ and the SM, while all SM fermions are neutral under $Z^{\prime}$. We classify the operators as a function of the gauge anomalies behaviour of mediators and explicitly compute the dimension-six operators coupling $Z^{\prime}$ to gluons, generated at one-loop by chiral but anomaly-free, sets of fermion mediators. We prove that only one operator contribute to the couplings between $Z^{\prime}$ charged matter and on-shell gluons. We then make a complete phenomenological analysis of the scenario where the lightest fermion charged under $Z^{\prime}$ is the dark matter candidate. Combining results from WMAP/PLANCK data, mono-jet searches at LHC, and direct/indirect dark matter detections restrict considerably the allowed parameter space.

KEYWORDS: Phenomenological Models

ARXIV EPRINT: 1307.0005 


\section{Contents}

1 Introduction and conclusions 1

$2 \quad Z^{\prime}$, heavy fermion mediators and effective operators $\quad 3$

2.1 Effective action from heavy fermion loops: coupling to gluons 6

2.2 "Anomalous" $Z^{\prime} \quad 8$

3 Dark matter annihilation to gluons $\quad 9$

3.1 The $s$-channel dark matter-gluons cross-section 10

$\begin{array}{lll}3.1 .1 & \text { Vector-coupling case } & 10\end{array}$

$\begin{array}{ll}\text { 3.1.2 Axial-vector couplings case } & 11\end{array}$

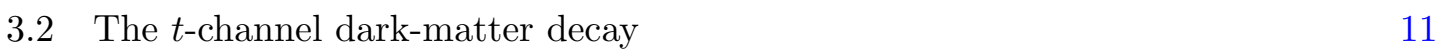

4 Experimental constraints $\quad 12$

$\begin{array}{lll}4.1 & \text { Relic abundance } & 12\end{array}$

$\begin{array}{lll}4.2 & \text { Indirect detection of dark matter } & 14\end{array}$

$\begin{array}{lll}4.3 & \text { Direct detection } & 15\end{array}$

$\begin{array}{lll}\text { 4.4 LHC analysis through mono-jets } & 15\end{array}$

$\begin{array}{ll}\text { 4.5 Constraints on the kinetic mixing } & 17\end{array}$

$\begin{array}{lll}4.6 & \text { Summary of the various constraints } & 18\end{array}$

$\begin{array}{lll}5 & Z^{\prime} \text { annihilation into electroweak gauge bosons } & 19\end{array}$

A Gauge independence and unitary gauge $\quad 22$

B Three-point gauge boson amplitude and gauge effective action from heavy fermion loops: couplings to gluons $\quad 24$

C Vanishing of the operator $\mathcal{T} r\left(F^{X} F_{\mathrm{SM}} \tilde{F}_{\mathrm{SM}}\right)$ and a useful identity 26

D The s and t-channel dark matter annihilation cross sections $\quad 27$

D.1 The s-channel electroweak annihilation cross sections into electroweak gauge $\begin{array}{ll}\text { bosons } & 27\end{array}$

D.2 The t-channel dark matter annihilation into $Z^{\prime} Z^{\prime} \quad 28$

\section{Introduction and conclusions}

New abelian gauge symmetries are arguably the simplest extensions of the Standard Model (SM) (for a recent review, see $[1,2]$ ) . If SM fermions are charged under a new abelian $\mathrm{U}(1)_{X}$, its couplings are strongly constrained by direct searches and especially by FCNC 
processes. The simplest and widely studied possibility in the literature is when SM fermions have flavor-independent charges. Most popular examples in this class are $B-L$ or linear combinations $\alpha(B-L)+\beta Y$. They are actually the only family-independent, anomaly-free gauged symmetries commuting with the SM gauge group in case where there are no new fermions charged under the Standard Model beyond the usual ones. Family-dependent anomaly-free models with no extra fermions were also extensively studied. ${ }^{1}$ In all such cases, the $Z^{\prime}$ should be heavy enough to escape detection, at least in the multi-TeV range. There is also a large literature on light U(1)'s of string or field theory origin with anomaly cancellation a la Green-Schwarz (for a very incomplete list of early papers, see [6-20, 79]), with low-energy anomalies canceled by axionic couplings and generalized Chern-Simons terms, or in other models with Stueckelberg realization of $Z^{\prime}$ [21-24].

A radically different option is to have no SM fermions charged under $Z^{\prime}$. This is a relatively natural framework in string theory with D-branes. But it is also natural from a field theory viewpoint, with additional heavy fermions $\Psi_{L, R}$, called "mediators" in what follows, which mediate effective interactions, described by the dimension-four kinetic mixing and higher-dimensional operators between the $Z^{\prime}$ and the SM sector [25, 26]. If one wants mediators parametrically heavier than the electroweak scale (say in the $\mathrm{TeV}$ range), we need, in addition to possible SM Higgs contributions, an additional source to their mass. A purely Dirac mass is of course a simple viable option. However as argued in [25, 26], because of the Furry theorem, the only low-dimensional induced effective operator is the kinetic mixing, whereas the next higher-dimensional ones are of dimension eight. Throughout our paper, we consider the kinetic mixing to be small enough. If we are interested in $Z^{\prime}$ couplings to gluons, this can be achieved for example by having colored mediators with no hypercharge. In this case, the main couplings between the "hidden" $Z^{\prime}$ and the SM are generated by higher-dimensional effective operators (hdo's), the lowest relevant ones being of dimension six. However, we will show that in the parameter space allowed by the PLANCK/WMAP data, the phenomenological consequences induced by the presence of a kinetic mixing allowed by various constraints are negligible. The simplest and natural option to obtain dimension-six effective operators is to generate the mediator masses by the vev of the scalar field $\phi$ breaking spontaneously the $Z^{\prime}$ gauge symmetry. The corresponding induced mediator masses, called generically $M$ in what follows, determine the mass scale of the hdo's and also the UV cutoff of the effective theory. There could also be contributions to their mass from the SM Higgs field $m \sim \lambda\langle H\rangle=\lambda v$, which are considered to be smaller, such that we can expand in powers of $v / M$ and obtain operators invariant under the SM gauge group. Such a framework was already investigated in [25-28] from the viewpoint of the effective couplings of $Z^{\prime}$ to electroweak gauge bosons. The potential implications to dark matter, considered to be the lightest fermion in the dark sector was also investigated, with the outcome that a monochromatic gamma ray line from the dark matter annihilation is potentially observable. The potential existence of a signal in the FERMI data was largely discussed in the recent literature ([30,31]; for a recent update on the prospects to confirm or to infirm this signature, see [32]) and will not be discussed further here.

\footnotetext{
${ }^{1}$ For recent updates on phenomenological and experimental constraints on such models, see e.g. [3-5].
} 
In this paper we extend the previous works by allowing the mediators to be colored and therefore the $Z^{\prime}$ to couple to gluons. We restrict ourselves throughout the paper to CP even couplings for simplicity. These couplings are more restricted by symmetries than the ones to the electroweak gauge bosons and their presence change significantly the phenomenology of such models. Whereas at dimension-six order four such operators are possible, only two of them are induced by heavy fermion mediators loops. Moreover, only one operator contributes to amplitudes in which at least one of the gluons is on-shell, as will be the case throughout our paper. We analyze in detail the corresponding phenomenology from the viewpoint of the dark matter relic abundance, direct and indirect dark matter detection and LHC constraints. Allowing couplings to gluons and at the same time to electroweak gauge bosons does not change significantly the phenomenology of the $Z^{\prime}$ compared to the case where only couplings to gluons are allowed. One interesting conceptual difference is that, whereas the $Z^{\prime}$ couplings to gluons and photons vanish for an on-shell $Z^{\prime}$ due to the Landau-Yang theorem [83, 84], the couplings to the electroweak gauge bosons $Z Z, Z \gamma$ do not vanish; they lead on the contrary to an enhancement close to the $Z^{\prime}$ pole. Another interesting result is that, unlike the case of kinetic mixing, the dark matter annihilation into gluons induced by virtual $Z^{\prime}$ exchange can give correct relic density for heavy dark matter and $Z^{\prime}$ masses, well above the electroweak scale. Since our interest here is to have complementary constraints from dark matter searches and LHC, we nonetheless confine our analysis to masses below than or of the order $\mathrm{TeV}$ in what follows.

The paper is organized as follows. Section 2 introduces the basic formalism we will use, which is Stueckelberg realization of $Z^{\prime}$ symmetry. It contains the list of the lowest dimensional effective operators generated by integrating-out heavy fermionic mediators, their classification depending on the nature of messenger masses and charges and the explicit loop computation of the $Z^{\prime}$ couplings to gluons. Section 3 deals with the consequences of the model for dark matter generation in the Early Universe, focusing on the annihilation to a gluon pair. Section 4 contains the various phenomenological constraints coming from the unique $Z^{\prime}$ coupling to gluons generated at one-loop by heavy colored mediators. Section 5 contains the re-analysis of the various constraints when $Z^{\prime}$ couplings to electroweak gauge bosons are also added. Appendices contain more details about the gauge independence of the $Z^{\prime}$ mediated hidden-sector-SM couplings, the effective operator couplings $Z^{\prime}$ to gluons induced by heavy mediator loops and the complete cross-sections of the s- and t-channel annihilation of the dark matter.

\section{$2 \quad Z^{\prime}$, heavy fermion mediators and effective operators}

The effective lagrangian generated by loops of heavy mediators is generically invariant under SM and has a non-linear (Stueckelberg) realization for $Z^{\prime}$, for the following reason. If the mediator masses are invariant under both the SM and the $Z^{\prime}$ gauge symmetry, the induced operators would be gauge invariant in the usual sense. If the mediator masses are however generated by the breaking of $\mathrm{U}(1)_{X}$, in the broken phase below the mass of the heavy Higgs $\phi$ breaking $\mathrm{U}(1)_{X}$, the symmetry is still present but realized a la Stueckelberg. Indeed, in the limit where $\phi$ is much heavier than the $Z^{\prime}$, in the effective theory we keep only the ax- 
ionic component of the original $Z^{\prime}$ Higgs field $\Phi=\frac{V+\phi}{\sqrt{2}} \exp \left(i a_{X} / V\right) \rightarrow \frac{V}{\sqrt{2}} \exp \left(i a_{X} / V\right)$. We define the dimensionless axion $\theta_{X}=\frac{a_{X}}{V}$ in what follows. The axion transforms non-linearly under $\mathrm{U}(1)$ transformations

$$
\delta Z_{\mu}^{\prime}=\partial_{\mu} \alpha \quad, \quad \delta \theta_{X}=\frac{g_{X}}{2} \alpha .
$$

The exact lagrangian, describing all the microscopic physics, including the mediator fields $\Psi_{L, R}$, is then of the form

$$
\begin{aligned}
\mathcal{L}= & \mathcal{L}_{\mathrm{SM}}+\bar{\Psi}_{L}^{i}\left(i \gamma^{\mu} D_{\mu}+\frac{g_{X}}{2} X_{L}^{i} \gamma^{\mu} Z_{\mu}^{\prime}\right) \Psi_{L}^{i}+\bar{\Psi}_{R}^{i}\left(i \gamma^{\mu} D_{\mu}+\frac{g_{X}}{2} X_{R}^{i} \gamma^{\mu} Z_{\mu}^{\prime}\right) \Psi_{R}^{i} \\
& -\left(\bar{\Psi}_{L}^{i} M_{i j} e^{\frac{i a_{X}\left(X_{L}^{i}-X_{R}^{j}\right)}{V}} \Psi_{R}^{i}+\text { h.c. }\right)+\frac{1}{2}\left(\partial_{\mu} a_{X}-M_{Z^{\prime}} Z_{\mu}^{\prime}\right)^{2}-\frac{1}{4} F_{\mu \nu}^{X} F^{X} \mu \nu
\end{aligned}
$$

where $\mathcal{L}_{\mathrm{SM}}$ is the Standard Model Lagrangian, $D_{\mu}$ 's are the covariant derivatives with respect to the standard model gauge group, and where $M_{Z^{\prime}}=g_{X} V / 2$. This lagrangian is indeed invariant under (2.1), with non-linear shifts of the axion $a_{X}$ crucial for restauring gauge invariance. If the original high-energy lagrangian is anomaly-free and the SM fermions are neutral under $Z^{\prime}$, then the mediators have to form an anomaly-free set. We are considering this class of models in most of this paper. In this case, the induced effective operators are gauge invariant a la Stueckelberg. Throughout the paper we restrict ourselves to $\mathrm{CP}$ even operators for simplicity. In the case where the mediators are not an anomaly-free set, then either low-energy fermions have to be charged under $Z^{\prime}$, or there are axionic couplings and GCS terms in order to cancel anomalies. ${ }^{2}$ For notational convenience we define:

$$
\begin{aligned}
D_{\mu} \theta_{X} & \equiv \partial_{\mu} \theta_{X}-\frac{g_{X}}{2} Z_{\mu}^{\prime}, & \widetilde{F}_{\mu \nu} & \equiv \frac{1}{2} \epsilon_{\mu \nu \rho \sigma} F^{\rho \sigma}, \\
\mathcal{T} r(F G) & \equiv \operatorname{Tr}\left[F_{\mu \nu} G^{\mu \nu}\right], & \mathcal{T} r(E F G) & \equiv \operatorname{Tr}\left[E_{\mu}{ }^{\lambda} F_{\lambda \nu} G^{\nu \mu}\right],
\end{aligned}
$$

where Tr takes into account a possible trace over non-abelian indices. In summary, there are three distinct possibilities:

i) The mediators are completely non-chiral, i.e. vector-like both respect to the SM and $\mathrm{U}(1)_{X}$. In this case, there are no dimension-six induced operators, since the only one that can be potentially written, $\mathcal{T} r\left(F^{X} F_{\mathrm{SM}} \tilde{F}_{\mathrm{SM}}\right)$ vanishes exactly as shown in the appendix.

ii) The mediators form an anomaly-free set, but are chiral with respect to $\mathrm{U}(1)_{X}$ and vector-like with respect to the SM. The induced dimension-six operators in this

\footnotetext{
${ }^{2} \mathrm{~A}$ general field-theoretical analysis with computation of these couplings and analysis of anomalies cancellation can be found in [10-12].
} 
case are

$$
\begin{aligned}
\mathcal{L}_{\mathrm{CP} \text { even }}^{(6)}= & \frac{1}{M^{2}}\left\{d_{g} \partial^{\mu} D_{\mu} \theta_{X} \mathcal{T} r(G \tilde{G})+d_{g}^{\prime} \partial^{\mu} D^{\nu} \theta_{X} \operatorname{Tr}\left(G_{\mu \rho} \tilde{G}_{\nu}^{\rho}\right)\right. \\
& \left.+e_{g} D^{\mu} \theta_{X} \operatorname{Tr}\left(G_{\nu \rho} \mathcal{D}_{\mu} \tilde{G}^{\rho \nu}\right)+e_{g}^{\prime} D_{\mu} \theta_{X} \operatorname{Tr}\left(G_{\alpha \nu} \mathcal{D}^{\nu} \tilde{G}^{\mu \alpha}\right)\right\} \\
+ & \frac{1}{M^{2}}\left\{D^{\mu} \theta_{X}\left[i\left(D^{\nu} H\right)^{\dagger}\left(c_{1} \tilde{F}_{\mu \nu}^{Y}+2 c_{2} \tilde{F}_{\mu \nu}^{W}\right) H+\text { h.c. }\right]\right. \\
& +\partial^{m} D_{m} \theta_{X}\left(d_{1} \mathcal{T} r\left(F^{Y} \tilde{F}^{Y}\right)+2 d_{2} \mathcal{T} r\left(F^{W} \tilde{F}^{W}\right)\right)+d_{e w}^{\prime} \partial^{\mu} D^{\nu} \theta_{X} \operatorname{Tr}\left(F_{\mu \rho} \tilde{F}_{\nu}^{\rho}\right) \\
& \left.+e_{e w} D^{\mu} \theta_{X} \operatorname{Tr}\left(F_{\nu \rho} \mathcal{D}_{\mu} \tilde{F}^{\rho \nu}\right)+e_{e w}^{\prime} D_{\mu} \theta_{X} \operatorname{Tr}\left(F_{\alpha \nu} \mathcal{D}^{\nu} \tilde{F}^{\mu \alpha}\right)\right\}
\end{aligned}
$$

where $\mathcal{D}_{\mu} G_{\alpha \beta}$ denotes the gluon covariant derivative, in components

$$
\mathcal{D}_{\mu} G_{\alpha \beta}^{a}=\partial_{\mu} G_{\alpha \beta}^{a}+g f^{a b c} G_{\mu}^{b} G_{\alpha \beta}^{c} .
$$

The last three terms in (2.4) refer to all electroweak gauge bosons.

iii) The mediators do not form an anomaly-free set. It means that some low-energy fermions have to be charged in order to compensate the resulting anomaly. The induced dimension-six operators in this case are not gauge invariant, but include axionic couplings and eventually GCS terms, schematically of the form

$$
\mathcal{L}=C_{i j}^{X} \frac{a_{X}}{V} \mathcal{T} r\left(F^{i} \tilde{F}^{j}\right)+E_{i j, k} \epsilon^{\mu \nu \rho \sigma} A_{\mu}^{i} A_{\nu}^{j} F_{\rho \sigma}^{k} .
$$

This case was studied from various perspectives in the past [10-12, 33-39] and will not be considered anymore here.

In all cases, there is potentially a kinetic mixing term [40, 41, 43, 44, 80]

$$
\frac{\delta}{2} F_{X}^{\mu \nu} F_{\mu \nu}^{Y}
$$

Mediators generate at one-loop $\delta \sim \frac{g_{X} g^{\prime}}{16 \pi^{2}} \sum_{i} X_{i} Y_{i} \ln \frac{\Lambda^{2}}{M_{i}^{2}}$, where $X_{i}, Y_{i}$ are the mediators charges to $\mathrm{U}(1)_{X}$ and $\mathrm{U}(1)_{Y}$, respectively. If $\delta$ has its natural one-loop value, then its effects are more important than most of the ones we will discuss in what follows. This is the most plausible case and was investigated in many details within the last years. In what follows, we will place ourselves in the mostly 'orthogonal' case in which $\delta$ is small enough such that its effects are subleading compared to the dimension-six operators. This is the case, for example, if messengers are in complete representations of a non-abelian gauge group (GUT groups are of course the best such candidates ${ }^{3}$ ), or if the mediators have no hypercharge.

Then, at low energy, the mediators being integrated out give rise to a new effective lagrangian

$$
\mathcal{L}_{\text {eff }}=\mathcal{L}_{1}\left(\psi^{\mathrm{DM}}, Z_{\mu}^{\prime}\right)+\mathcal{L}_{2}\left(A_{\mu}^{\mathrm{SM}}\right)+\mathcal{L}_{\text {mix }}\left(Z_{\mu}^{\prime}, A_{\mu}^{\mathrm{SM}}\right),
$$

\footnotetext{
${ }^{3}$ Assuming that there are no additional corrections to the kinetic mixing arising from heavy fields, like in the examples in $[45,46]$
} 
where $\mathcal{L}_{2}$ and $\mathcal{L}_{1}$ represent the new effective operators generated separately in the SM gauge sector and $Z^{\prime}$ one, whereas in $\mathcal{L}_{m i x}$ we collect all the induced terms mixing $Z^{\prime}$ with the Standard Model. Notice that $\mathcal{L}_{1}$ also contains the DM particle (i.e. the lightest mediator) which is not integrated out.

The mediators mass matrix has the symbolic form

$$
M_{i j}=\lambda_{i j} V+h_{i j} v,
$$

where $V$ is the vev breaking the $Z^{\prime}$ gauge group $\mathrm{U}(1)_{X}$ and $v$ is the electroweak vev. If the heavy Higgs $\phi$ has a charge 1, then the renormalizable Yukawas (2.9) exist provided

$$
\lambda_{i j} \neq 0\left(\text { and } h_{i j}=0\right) \quad \text { if } \quad X_{L}^{i}-X_{R}^{i}= \pm 1, \quad h_{i j} \neq 0\left(\text { and } \lambda_{i j}=0\right) \quad \text { if } \quad X_{L}^{i}-X_{R}^{i}=0 .
$$

Since none of our results in what follows depend on the assumption that the heavy fermions masses arise through renormalizable interactions, in the rest of the paper we include the more general case where these masses arise from arbitrary Yukawas of type

$$
\lambda_{i j} \Lambda(V / \Lambda)^{\left|X_{L}^{i}-X_{R}^{j}\right|} \bar{\Psi}_{L}^{i} \Psi_{R}^{j}+h . c
$$

where $\Lambda$ is an UV cut off, such that $\left|X_{L}^{i}-X_{R}^{j}\right|>1$ corresponds to non-renormalizable interactions. For phenomenological applications, we consider here a model in which the dark matter is represented by the lightest stable fermion $\psi^{\mathrm{DM}}$ charged under $Z^{\prime}$ and uncharged under SM (the mass of dark matter will be denoted by $m_{\psi}$ in what follows). The mediators $\Psi_{L, R}$ are considered to be heavy enough so that they have not been discovered yet in colliders. Assuming than they are heavier than both dark matter and the $Z^{\prime}$ boson, they can be integrated out so that we have to deal with effective operators, including new parameters. At the one-loop perturbative level, mediators generate only $Z^{\prime}$ couplings to the SM gauge fields and the SM Higgs as represented in figure 1 in the case of $Z^{\prime}$ coupling to gluons. Indeed, in the absence of kinetic mixing, one-loop couplings to SM fermions can be generated only if there are Yukawa couplings mixing mediators with SM fermions. We forbid such couplings in what follows. One (clearly not unique) way of achieving this is by defining a $Z_{2}$ parity, under which all mediator fields are odd and all SM fields are even.

In what follows we work in the unitary gauge where the axion is set to zero $\theta_{X}=0$. As usual, gauge invariance allows to work in any gauge. In the appendix we discuss the issue of gauge independence in more details.

\subsection{Effective action from heavy fermion loops: coupling to gluons}

In the case of exact $\mathrm{CP}$ invariance that we restrict for simplicity, the three-point gauge boson amplitude can be generally be written as [10-12]

$$
\begin{aligned}
\Gamma^{\mu \nu \rho}= & \epsilon^{\mu \nu \rho \alpha}\left(A_{1} k_{1 \alpha}+A_{2} k_{2 \alpha}\right) \\
& +\left[\epsilon^{\mu \nu \alpha \beta}\left(B_{1} k_{1}^{\rho}+B_{2} k_{2}^{\rho}\right)+\epsilon^{\mu \rho \alpha \beta}\left(B_{3} k_{1}^{\nu}+B_{4} k_{2}^{\nu}\right)\right] k_{1 \alpha} k_{2 \beta},
\end{aligned}
$$

where $A_{i}, B_{i}$ are Lorentz-invariant functions of the external momenta $k_{i}$. The functions $A_{i}$ which encode the generalized Chern-Simon terms (GCS) [10-12] are superficially logarithmically divergent, whereas the functions $B_{i}$ are UV finite. However, $A_{i}$ are determined in 

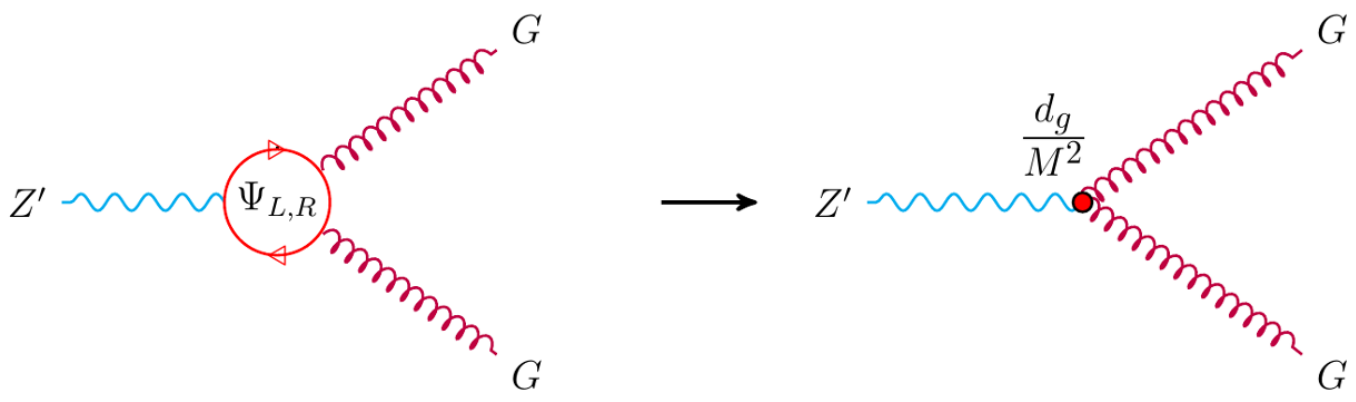

Figure 1. When heavy fermions are integrated out, they generate dimension-six effective operators of strength $d_{g} / M^{2}$.

terms of $B_{i}$ by using the Ward identities, which in the case where the heavy fermions form an anomaly-free set, are given by

$$
\begin{aligned}
k_{1}^{\nu} \Gamma_{\mu \nu \rho} & =0 \rightarrow A_{2}=B_{3} k_{1}^{2}+B_{4} k_{1} k_{2}, \\
k_{2}^{\rho} \Gamma_{\mu \nu \rho} & =0 \rightarrow A_{1}=B_{2} k_{2}^{2}+B_{1} k_{1} k_{2}, \\
-\left(k_{1}+k_{2}\right)^{\mu} \Gamma_{\mu \nu \rho} & =\left(A_{1}-A_{2}\right) \epsilon_{\nu \rho \alpha \beta} k_{1}^{\alpha} k_{2}^{\beta} \neq 0 .
\end{aligned}
$$

The violation of the $Z^{\prime}$ current conservation may seem surprising. It encodes actually the fact that one generates dimension-six operators, for which gauge invariance is realized à la Stueckelberg and indeed in the appendix B it will be shown explicitly that $A_{1} \neq A_{2}$. At the one loop order, there are several contributions to $\Gamma^{\mu \nu \rho}$. The first is the triangle loop diagram with no chirality flip/mass insertions, given by

$$
\Gamma_{\mu \nu \rho}^{(1)}=\sum_{i} t_{i a a} \int \frac{d^{4} p}{(2 \pi)^{4}} \operatorname{Tr}\left[\frac{\not p+\not \phi_{2}}{\left(p+k_{2}\right)^{2}-M_{i}^{2}} \gamma_{\rho} \frac{\not p}{p^{2}-M_{i}^{2}} \gamma_{\nu} \frac{\not p-\not k_{1}}{\left(p-k_{1}\right)^{2}-M_{i}^{2}} \gamma_{\mu} \gamma_{5}\right] .
$$

where $t_{i a a}=\operatorname{Tr}\left(X_{i} T^{a} T^{a}\right)$. As shown in the appendix B by using Ward identities, computing this diagram is enough in order to find the full amplitude. The final result for the $Z^{\prime}$ couplings and the details of the computation are described in the appendix B. After symmetrization among the two gluon legs, one finds

$\Gamma_{\mu \nu \rho}^{\mathcal{O}}=-\sum_{i} \frac{i t_{i a a, L-R}}{12 \pi^{2} M_{i}^{2}}\left\{\left[2\left(k_{1}+k_{2}\right)_{\mu} \epsilon_{\nu \rho \alpha \beta}-k_{1 \rho} \epsilon_{\mu \nu \alpha \beta}-k_{2 \nu} \epsilon_{\rho \mu \alpha \beta}\right] k_{1}^{\alpha} k_{2}^{\beta}+\epsilon_{\mu \nu \rho \alpha} k_{1} k_{2}\left(k_{2}-k_{1}\right)^{\alpha}\right\}$,

where $t_{i a a, L-R}=\operatorname{Tr}\left(\left(X_{L}-X_{R}\right) T^{a} T^{a}\right)_{i}$. The corresponding dimension-six operator for the triangle diagram represented in figure 2 is then

$$
\mathcal{O}=\frac{g_{3}^{2}}{24 \pi^{2}} \sum_{i} \operatorname{Tr}\left(\frac{\left(X_{L}-X_{R}\right) T_{a} T_{a}}{M^{2}}\right)_{i}\left[\partial^{\mu} D_{\mu} \theta_{X} \mathcal{T} r(G \tilde{G})-2 D_{\mu} \theta_{X} \operatorname{Tr}\left(G_{\alpha \nu} \mathcal{D}^{\nu} \tilde{G}^{\mu \alpha}\right)\right]
$$

where $g_{3}$ is the QCD strong coupling.

On the other hand, by using the identities (C.3) in appendix C, it can be shown that the antisymmetric part of the amplitude in the gluonic legs is zero, which is consistent 


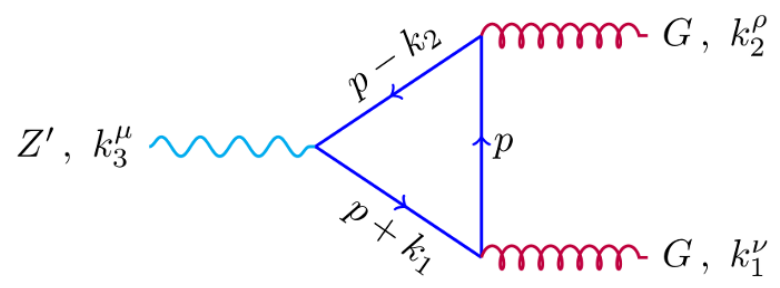

Figure 2. Integration of heavy fermions in a triangle diagram.

with the fact that there is no possible dimension-six operator mixing $Z^{\prime}$ to gluons, that is antisymmetric in the gluon fields. As a byproduct, we also find that the heavy mediators we are considering do not induce operators of the type

$$
\frac{1}{M^{2}} \operatorname{Tr}\left(G_{\mu \nu}\left[G^{\nu \lambda}, \tilde{G}_{\lambda}^{\mu}\right]\right)
$$

that are completely antisymmetric in the three gluon fields (2.15), and similar operators for electroweak gauge fields. This means that there are no constraints from purely SM dimension-six operators induced in this setup and all the phenomenological constraints come from the mixing of $Z^{\prime}$ with SM fields.

\section{2 "Anomalous" $Z^{\prime}$}

Until now we have made the important assumption that no SM fermion is charged under $Z^{\prime}$ and the only couplings arise through gauge-invariant higher-dimensional operators generated by integrating out heavy fermions forming an anomaly-free set. A more subtle option, in the spirit of $[10-12,16-20,25-28,79]$ is to integrate-out a set of heavy fermions which do contribute to gauge anomalies. In this case there are non-decoupling effects leading to axionic couplings and eventually generalized Chern-Simons terms. Let us consider two simple examples in order to exemplify the main points.

\section{i) Example with no colour anomalies:}

\begin{tabular}{|c|c|c|c|}
\hline Field & $Q_{3}^{L}$ & $t_{R}$ & $b_{R}$ \\
\hline$Z^{\prime}$ charge & 1 & 1 & 1 \\
\hline
\end{tabular}

In this case, after defining the anomaly coefficients $C_{a}=\operatorname{Tr}\left(X T_{a}^{2}\right)_{L-R}$ and $C_{X}=$ $\operatorname{Tr}\left(X^{2} Y\right)_{L-R}$, the low-energy effective theory has the following mixed anomalies:

$$
\begin{aligned}
& \mathrm{U}(1)_{X} \mathrm{SU}(3)^{2} \quad: \quad C_{3}=\frac{1}{2} \times(2-1-1)=0, \\
& \mathrm{U}(1)_{X} \mathrm{SU}(2)^{2} \quad: \quad C_{2}=\frac{1}{2} \times 3, \\
& \mathrm{U}(1)_{X} \mathrm{U}(1)_{Y}^{2} \quad: \quad C_{1}=6 \times \frac{1}{9}-3 \times\left(\frac{16}{9}+\frac{4}{9}\right)=-6, \\
& \mathrm{U}(1)_{X}^{2} \mathrm{U}(1)_{Y} \quad: \quad C_{X}=6 \times \frac{1}{3}-3 \times \frac{4}{3}+3 \times \frac{2}{3}=0 .
\end{aligned}
$$




\section{ii) Example with colour anomalies:}

\begin{tabular}{|c|c|c|c|}
\hline Field & $Q_{3}^{L}$ & $t_{R}$ & $b_{R}$ \\
\hline$Z^{\prime}$ charge & 1 & 1 & 0 \\
\hline
\end{tabular}

In this case, the low-energy effective theory has the following anomalies:

$$
\begin{aligned}
& \mathrm{U}(1)_{X} \mathrm{SU}(3)^{2}: \quad C_{3}=\frac{1}{2} \times(2-1)=\frac{1}{2}, \\
& \mathrm{U}(1)_{X} \mathrm{SU}(2)^{2}: \quad C_{2}=\frac{1}{2} \times 3, \\
& \mathrm{U}(1)_{X} \mathrm{U}(1)_{Y}^{2} \quad: \quad C_{1}=6 \times \frac{1}{9}-3 \times \frac{16}{9}=-\frac{14}{3}, \\
& \mathrm{U}(1)_{X}^{2} \mathrm{U}(1)_{Y} \quad: \quad C_{X}=6 \times \frac{1}{3}-3 \times \frac{4}{3}=-2 .
\end{aligned}
$$

In such examples, the heavy-fermion spectrum has to exactly cancel the low-energy gauge anomalies. In the decoupling limit there is an axionic coupling with a coefficient exactly determined by the low-energy induced anomalies

$$
\mathcal{L}_{\text {ax }}=\frac{a_{X}(x)}{16 \pi^{2} V}\left[\sum_{a}\left(C_{a} g_{a}^{2} \epsilon^{\mu \nu \rho \sigma} F_{\mu \nu}^{a} F_{\rho \sigma}^{a}\right)+C_{X} g_{X} g^{\prime} \epsilon^{\mu \nu \rho \sigma} F_{\mu \nu}^{X} F_{\rho \sigma}^{Y}\right] .
$$

As shown in the appendix B, we can also capture the effect of these axionic couplings in the unitary gauge, where the axionic effect is encoded in the particular high-energy behaviour of the anomalous three gauge boson amplitude with light fermions in the loop. This is strictly speaking true in the large (infinite) mass limit of heavy fermions. For finite mass, there are corrections and the low-energy description in the unitary gauge with three-gauge anomalous couplings is corrected by finite mass effects.

\section{Dark matter annihilation to gluons}

We start by first discussing the $Z^{\prime}$ couplings to gluons. The CP and gauge invariant dimension-six operators coupling $Z^{\prime}$ and the gluons are given by

$$
\begin{aligned}
\mathcal{L}_{\mathrm{CP} \text { even }}=\frac{1}{M^{2}}\left\{d_{g} \partial^{\mu} D_{\mu} \theta_{X} \mathcal{T} r(G \tilde{G})+d_{g}^{\prime} \partial^{\mu} D^{\nu} \theta_{X} \operatorname{Tr}\left(G_{\mu \rho} \tilde{G}_{\nu}^{\rho}\right)\right. \\
\left.\quad+e_{g} D^{\mu} \theta_{X} \operatorname{Tr}\left(G_{\nu \rho} \mathcal{D}_{\mu} \tilde{G}^{\rho \nu}\right)+e_{g}^{\prime} D_{\mu} \theta_{X} \operatorname{Tr}\left(G_{\alpha \nu} \mathcal{D}^{\nu} \tilde{G}^{\mu \alpha}\right)\right\} .
\end{aligned}
$$

Due to the fact that at one-loop only the operators with coeff. $d_{g}$ and $e_{g}^{\prime}$ are generated and only the first one contributes to the amplitude with on-shell gluons, we consider only $d_{g}$ in what follows and disregard the effects of the other operators in (3.1).

The dark matter couples minimally to the $Z^{\prime}$ boson as:

$$
\bar{\psi}_{L}^{\mathrm{DM}} \frac{g_{X}}{2} X_{L}^{\mathrm{DM}} \gamma^{\mu} Z_{\mu}^{\prime} \psi_{L}^{\mathrm{DM}}+\bar{\psi}_{R}^{\mathrm{DM}} \frac{g_{X}}{2} X_{R}^{\mathrm{DM}} \gamma^{\mu} Z_{\mu}^{\prime} \psi_{R}^{\mathrm{DM}},
$$

which provides us two ways of annihilating dark matter into gluons. The first one is an s-channel production of a $Z^{\prime}$ boson decaying into a pair of gluons. The second one is a 




(a)

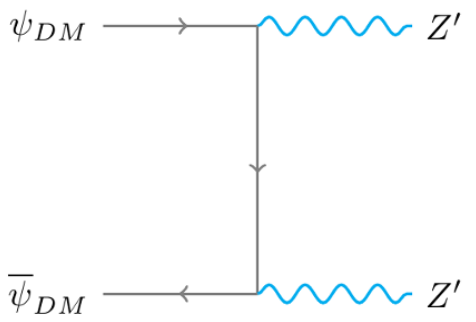

(b)

Figure 3. Gluon pair production via two different processes, that are the s-channel $(a)$ and the t-channel $Z^{\prime}$ pair production $(b)$, that decay subsequently into two gluons each.

t-channel process, leading to two $Z^{\prime}$ bosons, which will mostly decay into gluons. The associated Feynman diagrams are presented in figure 3.

In the unitary gauge, the $Z^{\prime}$-gluon-gluon vertex coming from the operator $d_{g}$ is

$$
\frac{d_{g}}{M^{2}}\left\{g_{X} \partial^{m} Z_{m}^{\prime} \epsilon^{\mu \nu \rho \sigma} \partial_{\mu} G_{\nu}^{A} \partial_{\rho} G_{\sigma}^{A}\right\}
$$

where the coeff. $d_{g}$ was redefined compared to (3.1) in a convenient way for our purposes.

The propagator of the vector boson $Z^{\prime}$ in the unitary gauge is

$$
\Delta(q)=-i \frac{\eta_{\mu \nu}-\frac{q_{\mu} q_{\nu}}{M_{Z^{\prime}}^{2}}}{q^{2}-M_{Z^{\prime}}^{2}+i M_{Z^{\prime}} \Gamma\left(Z^{\prime}\right)},
$$

For dark matter fermions of mass smaller than $M_{Z^{\prime}} / 2$, the main contribution to the $Z^{\prime}$ width $\Gamma\left(Z^{\prime}\right)$ is $\Gamma\left(Z^{\prime} \rightarrow \psi^{\mathrm{DM}} \overline{\psi^{\mathrm{DM}}}\right)$, which is computed to be

$$
\Gamma\left(Z^{\prime}\right)=\frac{g_{X}^{2}}{384 \pi M_{Z^{\prime}}^{2}}\left[\left(X_{L}^{2}+X_{R}^{2}\right) M_{Z^{\prime}}^{2}-\left(X_{L}^{2}+X_{R}^{2}-6 X_{R} X_{L}\right) m_{\psi}^{2}\right] \sqrt{M_{Z^{\prime}}^{2}-4 m_{\psi}^{2}} .
$$

For heavier masses of dark matter, one has to consider the $Z^{\prime}$ decay width into gluons and $\mathrm{SU}(2)$ gauge bosons. However, it can be readily checked that the detailed values of these widths do not influence much the results in what follows. ${ }^{4}$

\subsection{The $s$-channel dark matter-gluons cross-section}

\subsubsection{Vector-coupling case}

In the case of a vector-like coupling of DM fermion to $Z^{\prime}$ boson, one obtains the interaction lagrangian

$$
\mathcal{L}_{\text {int }}=\bar{\psi}^{\mathrm{DM}} \frac{g_{X}}{2} X^{\mathrm{DM}} \gamma^{\mu} Z_{\mu}^{\prime} \psi^{\mathrm{DM}}, \quad \text { where } \quad X^{\mathrm{DM}} \equiv X_{R}^{\mathrm{DM}}=X_{L}^{\mathrm{DM}}
$$

\footnotetext{
${ }^{4}$ Indeed, we will see in what follows that the cross section of dark matter annihilation into gluons is suppressed for an invariant mass $\sqrt{s}$ approaching $M_{Z^{\prime}}$, as a consequence of the Landau-Yang theorem [83, 84]. In the non-relativistic approximation, this happens in the energy region closed to $s \simeq 4 m_{\psi}^{2}+m_{\psi}^{2} v_{r e l}^{2} \geqslant$ $4 m_{\psi}^{2}$. The suppression therefore occurs for a mass $m_{\psi}$ significantly lower than $M_{Z^{\prime}} / 2$, where the decay width is essentially that of decay into two dark matter particles.
} 
Now we can perform the tree-level diagram cross section. We find that the amplitude vanishes $\mathcal{M}=0$ and therefore the $d_{g}$-term does not contribute to the final cross section at all. The reason is that, due to the effective coupling of the form $d_{g} \partial^{m} Z_{m}^{\prime} \mathcal{T} r(G \tilde{G})$, the vertex $Z^{\prime} \psi^{\mathrm{DM}} \psi^{\mathrm{DM}}$ gets multiplied by the virtual momentum and is of the form

$$
q^{\mu} \bar{v}\left(p_{2}\right) \gamma_{\mu} u\left(p_{1}\right)=\bar{v}\left(p_{2}\right)\left(\not \not p_{2}+\not \not_{1}\right) u\left(p_{1}\right)=0
$$

after using Dirac equation for the spinors describing the wavefunctions of the dark matter fermions.

\subsubsection{Axial-vector couplings case}

In the general case we get also an axial-vector coupling in addition to the vector one

$$
\mathcal{L}_{\text {int }}=\frac{g_{X}}{2}\left(\frac{X_{R}^{\mathrm{DM}}+X_{L}^{\mathrm{DM}}}{2}\right) \bar{\psi}^{\mathrm{DM}} \gamma^{\mu} Z_{\mu}^{\prime} \psi^{\mathrm{DM}}+\frac{g_{X}}{2}\left(\frac{X_{R}^{\mathrm{DM}}-X_{L}^{\mathrm{DM}}}{2}\right) \bar{\psi}^{\mathrm{DM}} \gamma^{\mu} \gamma_{5} Z_{\mu}^{\prime} \psi^{\mathrm{DM}}
$$

One then gets, as far as the annihilation of dark matter into a gluon pair is concerned, the total cross section

$$
\sigma_{\mathrm{s}-\text { channel }}\left(\psi^{\mathrm{DM}} \psi^{\mathrm{DM}} \rightarrow G G\right)=\frac{d_{g}^{2}}{M^{4}} \frac{\left(-4 E^{2}+M_{Z^{\prime}}^{2}\right)^{2}}{\left(-4 E^{2}+M_{Z^{\prime}}^{2}\right)^{2}+M_{Z^{\prime}}^{2} \Gamma^{2}\left(Z^{\prime}\right)} \frac{E^{5} g_{X}^{4} m_{\psi}^{2}\left(X_{L}-X_{R}\right)^{2}}{\pi M_{Z^{\prime}}^{4} \sqrt{E^{2}-m_{\psi}^{2}}} .
$$

The cross section is suppressed for energies of order $M_{Z^{\prime}} / 2$ due to the Landau-Yang theorem. There is also a helicity suppression for light dark matter case, that can be easily understood by writing the vertex $Z^{\prime} \psi^{\mathrm{DM}} \psi^{\mathrm{DM}}$ in this case

$$
\begin{aligned}
\left(X_{L}-X_{R}\right) q^{\mu} \bar{v}\left(p_{2}\right) \gamma_{\mu} \gamma_{5} u\left(p_{1}\right) & =\left(X_{L}-X_{R}\right) \bar{v}\left(p_{2}\right)\left(\not p_{2} \gamma_{5}-\gamma_{5} p_{1}\right) u\left(p_{1}\right) \\
& =-2 m_{\psi}\left(X_{L}-X_{R}\right) \bar{v}\left(p_{2}\right) \gamma_{5} u\left(p_{1}\right),
\end{aligned}
$$

after using Dirac equation.

This finally gives in the non-relativistic approximation $s \simeq 4 m_{\psi}^{2}+m_{\psi}^{2} v_{r e l}^{2} \Leftrightarrow E \simeq$ $m_{\psi} \sqrt{1+\frac{v_{r e l}^{2}}{4}}$, with $v_{r e l}$ being the relative velocity between the two colliding dark matter fermions, the total cross section

$$
\langle\sigma v\rangle_{\mathrm{s}-\text { channel }} \simeq \frac{d_{g}^{2}}{M^{4}} \frac{g_{X}^{4} m_{\psi}^{6}\left(X_{L}-X_{R}\right)^{2}}{\pi M_{Z^{\prime}}^{4}}\left\{\frac{2\left(M_{Z^{\prime}}^{2}-4 m_{\psi}^{2}\right)^{2}}{\left(M_{Z^{\prime}}^{2} \Gamma^{2}\left(Z^{\prime}\right)+\left(M_{Z^{\prime}}^{2}-4 m_{\psi}^{2}\right)^{2}\right)}\right\}+\mathcal{O}\left(v^{2}\right)
$$

\subsection{The $t$-channel dark-matter decay}

As mentioned earlier, we also have to consider a t-channel process, producing pairs of $Z^{\prime}$ bosons in dark matter annihilation for $Z^{\prime}$ mass below the dark matter mass. Considering that the only non vanishing coupling is the one in $d_{g}$, each $Z^{\prime}$ will decay into gluons; this process will then produce gluons in the final state. After expanding in powers of $v^{2}$, the 
cross-section in this case can be expressed as:

$$
\begin{gathered}
\langle\sigma v\rangle_{\mathrm{t}-\text { channel }}=\frac{g_{X}^{4} \sqrt{m_{\psi}^{2}-M_{Z^{\prime}}^{2}}}{128 \pi^{2} m_{\psi} M_{Z^{\prime}}^{2}\left(2 m_{\psi}^{2}-M_{Z^{\prime}}^{2}\right)^{2}} \\
\times\left(2 m_{\psi}^{4} X_{L}^{4}-4 m_{\psi}^{4} X_{L}^{2} X_{R}^{2}+2 m_{\psi}^{4} X_{R}^{4}-3 m_{\psi}^{2} M_{Z^{\prime}}^{2} X_{L}^{4}\right. \\
+10 m_{\psi}^{2} M_{Z^{\prime}}^{2} X_{L}^{2} X_{R}^{2}-3 m_{\psi}^{2} M_{Z^{\prime}}^{2} X_{R}^{4}+M_{Z^{\prime}}^{4} X_{L}^{4} \\
\left.-6 M_{Z^{\prime}}^{4} X_{L}^{2} X_{R}^{2}+M_{Z^{\prime}}^{4} X_{R}^{4}\right)+\mathcal{O}\left(v^{2}\right) .
\end{gathered}
$$

\section{Experimental constraints}

A $Z^{\prime} G G$ coupling can be tested in several laboratories, from direct detection experiments to indirect detection, relic abundance or LHC searches. We present in the following the constraints obtained from these different searches, before summarizing all of them at the end of the section. The reader can also find a nice recent complementary analysis of gluonic effective couplings to dark matter in [50].

\subsection{Relic abundance}

Recently, PLANCK collaboration released its latest results concerning the composition of the Universe [51]. It confirms the results of WMAP experiment [52] obtaining for the relic abundance of non-baryonic matter $\Omega h^{2}=0.1199 \pm 0.0027$ at $68 \%$ of CL. With such a level of precision, it is interesting to know what is the effective scale $M$ which is able to produce sufficient dark matter from the thermal bath to respect the previous PLANCK/WMAP results. Depending on the spectrum, two annihilation processes allow the dark matter candidate to keep thermal equlibrium with the standard model particles of the plasma: the $s$-channel exchange of a $Z^{\prime}$ (eq. (3.11)), and the $t$-channel production of the $Z^{\prime}$ (eq. (3.12)), as long as this channel is kinematically open.

Concerning the numerical analysis, we solved the Boltzmann equations by developing a code and adapting the public software MicrOMEGAs [47, 53, 54] to our model. We then extracted the relic abundance and checked that our analytical solutions (3.11)-(3.12) gives similar results to the numerical procedure ${ }^{5}$ at a level of 20 to $30 \%$. We noticed in section 3.1.1 that the coupling of the dark matter should be axial, as the vectorial part of the current coupling to $Z_{\mu}^{\prime}$ does not gives any contribution to the process $\psi^{\mathrm{DM}} \psi^{\mathrm{DM}} \rightarrow$ $Z^{\prime} \rightarrow G G$. For simplicity, we will set charges $X_{R}=1, X_{L}=2 \Rightarrow\left|X_{R}-X_{L}\right|=1$. Our results for a different set of charges are modified in a straightforward way. To keep our results as conservative as possible, we plotted the WMAP limits $0.087<\Omega h^{2}<0.138$ at $5 \sigma$.

We show in figure 4 the parameter space allowed in the plane $\left(\frac{M^{2}}{d_{g}}, m_{\psi}\right)$ for different values of $M_{Z^{\prime}}$ and $g_{X}$. Points above the red lines region would lead to an overpopulation of

\footnotetext{
${ }^{5}$ Mainly because the dominant annihilations are dominated by s-wave processes and the solution $\langle\sigma v\rangle \simeq$ $3 \times 10^{-26} \mathrm{~cm}^{3} \mathrm{~s}^{-1} \simeq 2.5 \times 10^{-9} \mathrm{GeV}^{-2}$ gives reasonable good approximations to the full Boltzmann system of equations.
} 

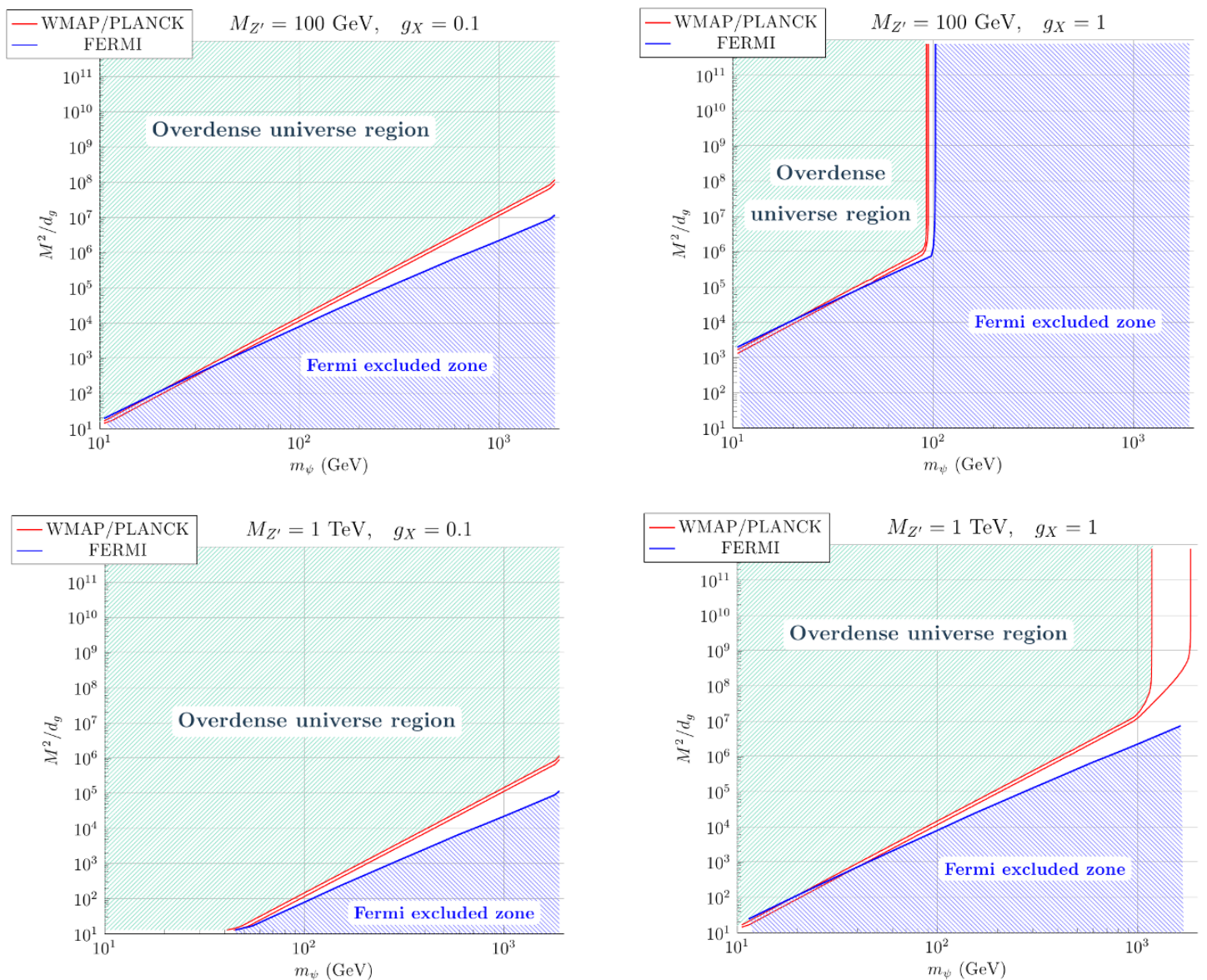

Figure 4. Constraints from WMAP/PLANCK (red line) and FERMI dSphs galaxies (blue line) in the $\left(\frac{M^{2}}{d_{g}}, m_{\psi}\right)$ plane for different values of $g_{X}\left(0.1\right.$ on the left and 1 on the right), $M_{Z^{\prime}}=100 \mathrm{GeV}$ (up) and $M_{Z^{\prime}}=1 \mathrm{TeV}$ (down). See the text for more details.

dark matter whereas points lying below the red lines would require additional dark matter candidates to respect PLANCK/WMAP constraints. We can notice several, interesting features from these results. First of all, we observe that as soon as the $Z^{\prime} Z^{\prime}$ final state is kinematically allowed $\left(m_{\psi}>M_{Z^{\prime}}\right)$ this annihilation channel is the dominant one as soon as $g_{X}$ is sufficiently large (we checked that this happens for $g_{X} \gtrsim 0.3$ ) and mainly independent on the dark matter mass. This is easy to understand after an inspection of eq. (3.12). Indeed, in the limit $m_{\psi} \gg M_{Z^{\prime}}$, one obtains $\langle\sigma v\rangle_{Z^{\prime} Z^{\prime}} \simeq \frac{9 g_{X}^{4}}{256 \pi^{2} M_{Z^{\prime}}^{2}}$. In other words, once

$$
\frac{9 g_{X}^{4}}{256 \pi^{2} M_{Z^{\prime}}^{2}} \gtrsim 2.5 \times 10^{-9} \mathrm{GeV}^{-2} \rightarrow g_{X} \gtrsim 3 \times 10^{-2} \sqrt{\frac{M_{Z^{\prime}}}{\mathrm{GeV}}}
$$

then the $t$-channel process $\psi^{\mathrm{DM}} \psi^{\mathrm{DM}} \rightarrow Z^{\prime} Z^{\prime}$ dominates the annihilation and forbids the dark matter to overpopulate of the Universe $\left(\Omega_{\psi} h^{2} \lesssim 0.138\right)$. This corresponds to $g_{X} \simeq 0.3$ for $M_{Z^{\prime}}=100 \mathrm{GeV}$ and $g_{X} \simeq 1$ for $M_{Z^{\prime}}=1 \mathrm{TeV}$, which fits pretty accurately the numerical results we obtained. This limit also explains why the region allowed by PLANCK/WMAP 
is larger for $M_{Z^{\prime}}=1 \mathrm{TeV}$ : the value $g_{X}=1$ is at the border limit for the $t$-channel to dominate. From eq. (4.1) we also understand why the $Z^{\prime} Z^{\prime}$ final state, even if kinematically allowed, has no influence on the limits set by the relic abundance for $g_{X}=0.1$ : the coupling is too small to give sufficient annihilation products. The dominant process is then the $s$-channel $Z^{\prime}$ exchange ( $\simeq 15 \%$ of $Z^{\prime} Z^{\prime}$ final state for $g_{X}=0.1$ and $M_{Z^{\prime}}=1 \mathrm{TeV}$.).

A different choice for the charges $X_{L}$ and $X_{R}$ has a straightforward influence on this result since it will change an overall factor in eq. (4.1). As an example, taking $X_{R}=5$ and $X_{L}=6$ will give

$$
\langle\sigma v\rangle_{Z^{\prime} Z^{\prime}} \simeq \frac{121 g_{X}^{4}}{256 \pi^{2} M_{Z^{\prime}}^{2}} \gtrsim 2.5 \times 10^{-9} \mathrm{GeV}^{-2} \rightarrow g_{X} \gtrsim 1.5 \times 10^{-2} \sqrt{\frac{M_{Z^{\prime}}}{\mathrm{GeV}}}
$$

implying that the t-channel will become dominant for $g_{X} \simeq 0.1$ for $M_{Z^{\prime}}=100 \mathrm{GeV}$ and $g_{X} \simeq 0.4$ for $M_{Z^{\prime}}=1 \mathrm{TeV}$. The parameter space will then be slightly enlarged.

We also notice in figure 4 that the region of the parameter space respecting WMAP/PLANCK data with a dominant $s$-channel annihilation seems linear (in logarithmic scale). This can be easily understood; indeed, after a glance at eq. (3.11), one obtains $^{6}$

$$
\langle\sigma v\rangle \simeq \frac{d_{g}^{2}}{M^{4}} \frac{2 g_{X}^{4}}{\pi} \frac{m_{\psi}^{6}}{M_{Z^{\prime}}^{4}} \quad\left(\text { for } M_{Z^{\prime}} \gg m_{\psi} \text { or } \quad M_{Z^{\prime}} \ll m_{\psi}\right),
$$

which imply for constant $\langle\sigma v\rangle$,

$$
\log \left(\frac{M^{2}}{d_{g}}\right)=3 \log m_{\psi}+\text { const }
$$

which is exactly the behavior we observe in figure 4 .

\subsection{Indirect detection of dark matter}

Other astrophysical constraints arise from the diffuse gamma ray produced by the dark matter annihilation in the center of Milky Way [55], the galactic halo [56], the dwarf spheroidal galaxies [57-59] or the radio observation of nearby galaxies like M31 [60-62]. Even if the authors of [60-62] claimed that their limits "exceed the best up-to-day known constraints from Fermi gamma observations", the dependence on magnetic fields profiles and charged particles propagation in M31 medium brings some uncertainties difficult to evaluate. The same remark is valid for the galactic center study [55] where the region of the sky and the cut made to analyze the data depends strongly on the dark matter halo profile in play to maximize the signal/background ratio. We will then consider the more reliable constraints obtained by the observation of dwarf galaxies by the FERMI telescope [5759]. These galaxies being mainly composed of dark matter, the background is naturally minimized.

We show the result of our analysis in figure 4 where the points below and on the right of the blue lines are excluded by FERMI observations. As expected, the region below $m_{\psi} \lesssim 40-50 \mathrm{GeV}$ (where the curves from FERMI and WMAP/PLANCK cross) is in

\footnotetext{
${ }^{6}$ Neglecting the tiny region around the pole $M_{Z^{\prime}}=2 m_{\psi}$.
} 
tension with FERMI limit, as hadronic final states are the more restricted by FERMI analysis $^{7}$, which seems to exclude any thermal relics below this dark matter mass. When the $Z^{\prime} Z^{\prime}$ final state is allowed, the annihilation cross section $\psi \psi \rightarrow Z^{\prime} Z^{\prime}$ is so large that is is almost automatically excluded by FERMI data.

\subsection{Direct detection}

For direct detection purposes, one can integrate out the $Z^{\prime}$ gauge boson and write the corresponding dimension-eight operator connecting the dark matter with the gluons. One gets

$$
\frac{d_{g}}{M^{2} M_{Z^{\prime}}^{2}} \bar{\psi}^{\mathrm{DM}} \gamma^{\mu}\left(\frac{X_{R}+X_{L}}{2}+\frac{X_{R}-X_{L}}{2} \gamma_{5}\right) \psi^{\mathrm{DM}} \mathcal{T} r \partial_{\mu}(G \tilde{G})
$$

By using the observed CP invariance of the strong interactions, we find that the only non-vanishing relevant gluonic matrix element we can write between an initial and a final nucleon state is $\left\langle N(p)\left|\operatorname{Tr} G_{\mu}^{\nu} \tilde{G}_{\nu}^{\lambda}\right| N\left(p^{\prime}\right)\right\rangle=A \epsilon_{\mu}^{\lambda \alpha \beta} p_{\alpha} p_{\beta}^{\prime}$, where $A$ is a Lorentz invariant. As a consequence,

$$
\left\langle N(p)\left|\mathcal{T} r \partial_{\mu}(G \tilde{G})\right| N\left(p^{\prime}\right)\right\rangle=0 .
$$

There are therefore no constraints on this operator from direct detection experiments.

\subsection{LHC analysis through mono-jets}

The model described in previous sections can be probed at the LHC. Indeed the $Z^{\prime}$-gluongluon vertex makes possible to produce a dark matter pair out of two protons, provided a $Z^{\prime}$ is produced. Typical production channels are shown in figure 5 , where we consider a generic process:

$$
p p \rightarrow j \bar{\psi}_{\mathrm{DM}} \psi_{\mathrm{DM}}
$$

of a proton-proton collision giving rise to 1 jet, plus missing energy $\left(E_{T}^{\text {miss }}\right)$.

The monojet final state was first studied using Tevatron data [63] in the framework of effective $\psi_{\mathrm{DM}}$-quark interactions of different nature. In a similar fashion, bounds to dark matter effective models have been obtained by analyzing single-photon final states using LEP [64] and LHC [65] data. An interesting complementarity between these two approaches has been analyzed in [66]. Since then, the ATLAS and CMS groups have taken the mono-signal analyses as an important direction in the search for dark matter at the LHC (see [67] and [68] for the most recent results from ATLAS and CMS, respectively). The most important background to the dark matter signal is coming from the Standard Model production of a $Z$ boson decaying to a neutrino pair $(Z \rightarrow \bar{\nu} \nu)$, however, in the inclusive analysis other processes like $W \rightarrow \ell \nu$ are considered as well. Other interesting and solid studies can be found in [69-71].

In this paper we use the monojet data coming from the CMS analysis [68], which collected events using a center-of-mass energy of $8 \mathrm{TeV}$ up to an integrated luminosity of $19.5 / \mathrm{fb}$. We perform the analysis by looking at the distribution of the jet's transverse

\footnotetext{
${ }^{7}$ Notice however that FERMI considers in their analysis the $Z^{\prime}$ decays into quarks, whereas in our case it decays into gluons.
} 

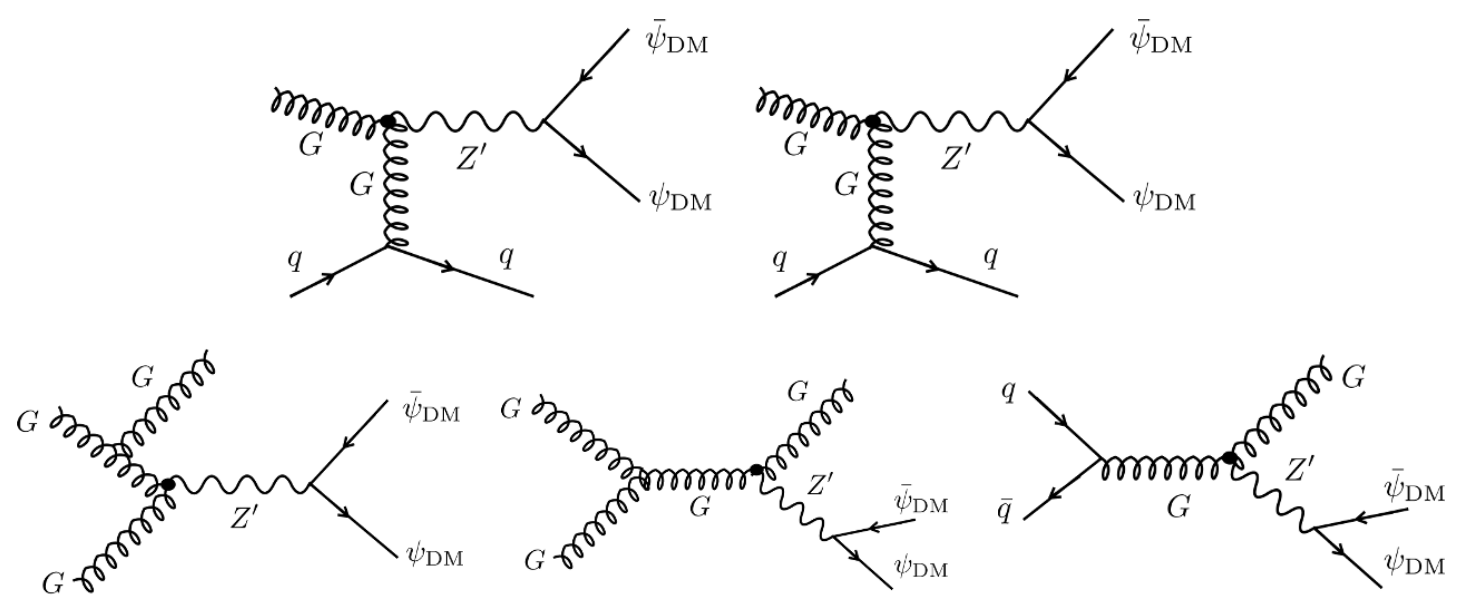

Figure 5. Dark matter production processes at the LHC (at partonic level), in association with 1 jet: $p p \rightarrow j \bar{\psi}_{\mathrm{DM}} \psi_{\mathrm{DM}}$.

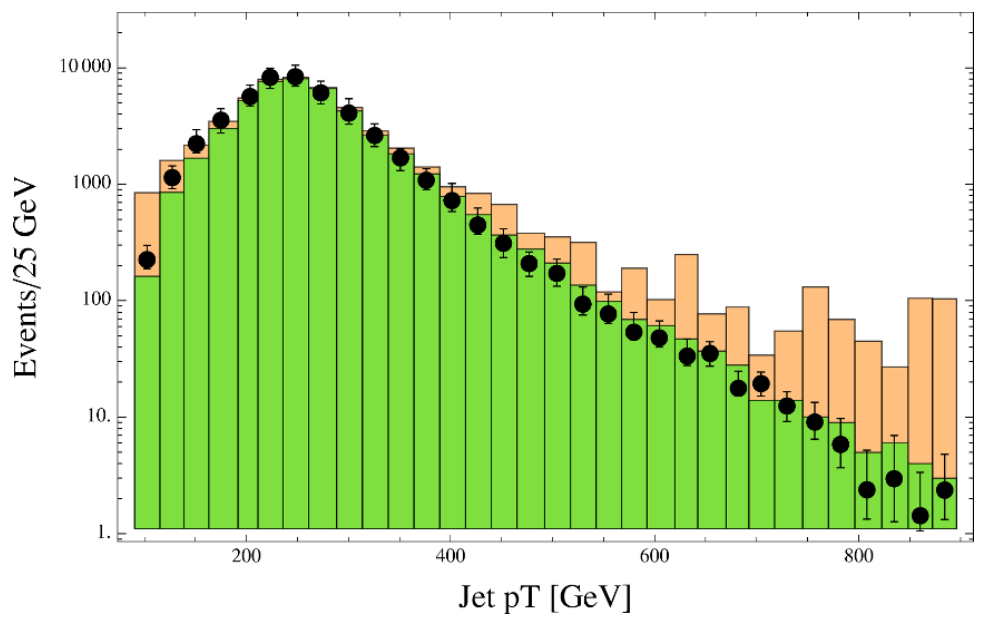

Figure 6. Histogram of $p_{T}^{\text {jet }}$ corresponding to a particular choice of the model parameters (see text for details). The signal is shown in orange. The background (green bars) and data (points) are taken from the CMS analysis.

momentum $\left(p_{T}^{\text {jet }}\right)$, taking the background analysis given in [68] and simulating on top the signal coming from our model. For the event generation we use CalcHEP.3.4.2 [72].

A typical histogram is shown in figure 6 , where we have used $m_{\psi}=10 \mathrm{GeV}, M_{Z^{\prime}}=$ $100 \mathrm{GeV}$ and $^{8} d_{g} / M^{2}=10^{-6}$ as the model parameters.

The results are shown in figure 7, where we show the exclusion power of the monojet analysis to the model. We present the bounds for the quantity $M^{2} / d_{g}$ as a function of the dark matter mass, for three different values of the $Z^{\prime}$ mass: $100 \mathrm{GeV}, 500 \mathrm{GeV}$ and $1 \mathrm{TeV}$.

The shape and relative size of the bounds can be understood by looking at the amplitude of the processes, which are proportional to $c^{2} m_{\psi}^{2} / M_{Z^{\prime}}^{4}$, where the coupling $c \equiv d_{g} / M^{2}$. For example, given a $M_{Z}^{\prime}$, for $m_{\psi}=10 \mathrm{GeV}$ the bounds are approximately 10 times weaker

\footnotetext{
${ }^{8}$ We took for the figure the illustrative case $\left|X_{L}-X_{R}\right| g_{X}^{2}=1$. Results other values of the coupling are obtained by a simple rescaling of the number of events.
} 


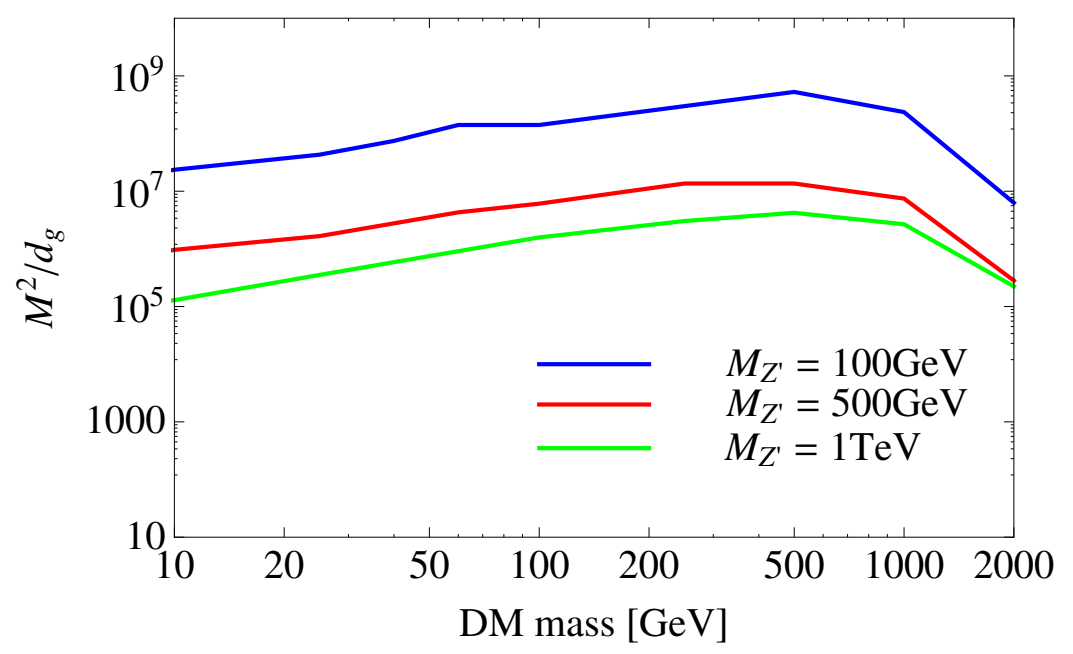

Figure 7. $90 \%$ CL lower bounds on the quantity $M^{2} / d_{g}$ as a function of the dark matter mass, for $M_{Z^{\prime}}=100 \mathrm{GeV}$ (blue), $500 \mathrm{GeV}$ (red) and $1 \mathrm{TeV}$ (green). Based on the CMS analysis with collected data using a center-of-mass energy of $8 \mathrm{TeV}$ and a luminosity of 19.5/fb.

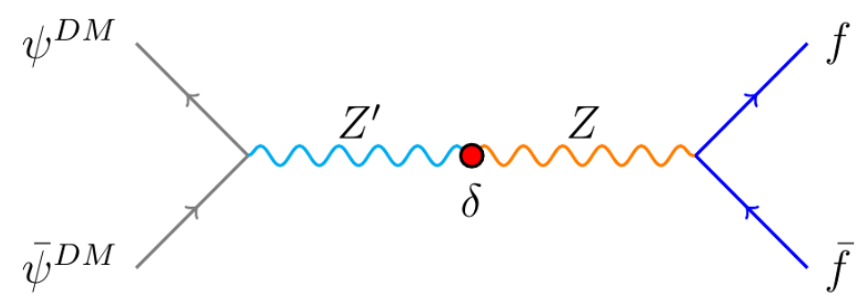

Figure 8. Example of $f \bar{f}$ production, from a dark matter annihilation and via an $s$-channel $Z / Z^{\prime}$ exchange.

than those for $m_{\psi}=100 \mathrm{GeV}$. However, for $m_{\psi} \gtrsim 1 \mathrm{TeV}$ the dark matter starts to be too heavy to be easily produced out of the $4 \mathrm{TeV}$ protons, given the PDF suppression of the quarks and gluons; so the DM production is close to be kinematically closed. On the other hand, for example at $m_{\psi}=100 \mathrm{GeV}$, the bound for $M_{Z^{\prime}}=100 \mathrm{GeV}$ is around 25 (100) times stronger than the one corresponding to $M_{Z^{\prime}}=500(1000) \mathrm{GeV}$.

\subsection{Constraints on the kinetic mixing}

All through the analyses we considered a small kinetic mixing. However it can be interesting to check to what extent this hypothesis is valid. Indeed, whereas it exists various constraints $^{9}$ on $\delta$ (from precision measurements, rare decay processes, $\rho$-parameter), a nonzero kinetic mixing generates new annihilation diagrams ( $s$-channel $Z / Z^{\prime}$ exchange), as represented in figure 8 , which could modify our results. ${ }^{10}$

To test the validity of our approach, we extract from eq. (3.11) an approximate solution for the gluonic annihilation cross section (we ignore here the factors of $X_{L}-X_{R}$

\footnotetext{
${ }^{9}$ The literature on the subject is very vast. We suggest for further reading [73-77] for dark matter constraints, [78] for LHC constraints, [79, 80] for string motivations and [81, 82] for other studies.

${ }^{10}$ In all our study we use the conventions described in [76, 77].
} 
for simplicity):

$$
\langle\sigma v\rangle_{G G} \simeq \frac{d_{g}^{2}}{M^{4}} \frac{2 g_{X}^{4}}{\pi} \frac{m_{\psi}^{6}}{M_{Z^{\prime}}^{4}}
$$

Concerning the annihilation generated by the $s$-channel exchange of a $Z / Z^{\prime}$ through kinetic mixings (see figure 8), the expressions of the cross section can be found in [86] and approximated by: ${ }^{11}$

$$
\begin{aligned}
\langle\sigma v\rangle_{\delta} & \simeq \frac{16}{\pi} g_{X}^{2} g^{2} \delta^{2} \frac{m_{\psi}^{2}}{M_{Z^{\prime}}^{4}}, & & m_{\psi}<M_{Z} \\
\langle\sigma v\rangle_{\delta} & \simeq \frac{g_{X}^{2} g^{2} \delta^{2} M_{Z}^{4}}{\pi m_{\psi}^{2} M_{Z^{\prime}}^{4}}, & & m_{\psi}>M_{Z} .
\end{aligned}
$$

We can then obtain the value of $\delta$ for which the process $\langle\sigma v\rangle_{\delta}$ dominates on $\langle\sigma v\rangle_{G G}$, invalidating our analysis done by ignoring the kinetic mixing:

$$
\begin{array}{ll}
\delta \gtrsim \frac{d_{g}}{M^{2}} \frac{g_{X}}{2 \sqrt{2} g} m_{\psi}^{2}, & m_{\psi}<M_{Z} \\
\delta \gtrsim \frac{d_{g}}{M^{2}} \frac{\sqrt{2} g_{X}}{g} \frac{m_{\psi}^{4}}{M_{Z}^{2}}, & m_{\psi}>M_{Z}
\end{array}
$$

which give for example for $m_{\psi}=200 \mathrm{GeV}$ and $g_{X}=0.1, \frac{M^{2}}{d_{g}} \gtrsim \frac{10^{4}}{\delta} \mathrm{GeV}^{2}$. In other words, for values of the coupling $\frac{d_{g}}{M^{2}} \lesssim 10^{-4} \times \delta \mathrm{GeV}^{-2}$, the annihilation processes induced by kinetic mixing begin to compete with the gluonic final state. Another interesting point is that the conditions are independent on the mass of the $Z^{\prime}$ as soon as we assume $M_{Z^{\prime}} \gg M_{Z}$.

To confirm our conclusions, we made a numerical analysis, allowing a non-zero kinetic mixing. We show in figure 9 the iso-curve for the branching ratio $\langle\sigma v\rangle_{\psi \psi \rightarrow G G}$ in the plane $\left(\delta ; d_{g} / M^{2}\right)$ given by our numerical analysis. We also draw the region allowed by WMAP at $5 \sigma .{ }^{12}$ We took $M_{Z^{\prime}}=1 \mathrm{TeV}, m_{\psi}=200 \mathrm{GeV}$ and $g_{X}=0.1$ but we checked that the result is generic for broad regions in the parameter space. ${ }^{13}$ We first notice that the region respecting the cosmological bounds lie in a region where the gluonic fraction is largely dominant (over 90\%). It is only for very high values of $\delta \simeq 0.8$ that the channel $\psi \psi \rightarrow Z / Z^{\prime} \rightarrow$ SM SM can contribute at a substantial level $(\simeq 10 \%)$ to the relic density computation, confirming with a surprising accuracy our analytic results eq. (4.10). Such values for $\delta$ are already excluded by LEP experiments.

\subsection{Summary of the various constraints}

Now we can put together all the constraints we obtained on the parameter pair $\left(m_{\psi}, \frac{M^{2}}{d_{g}}\right)$ to see what are the new allowed regions in the parameter space. Superposing figure 4 and 7 , we get a new representation of those validity zones, as represented in figure 10 .

\footnotetext{
${ }^{11}$ These expressions are valid in the regime $M_{Z^{\prime}}>M_{Z}$ but a similar analysis can be performed in the case $M_{Z^{\prime}}<M_{Z}$.

${ }^{12}$ The WMAP constraint is quite insensitive to $\delta$ in the range of values shown in figure 9 , however for large $\delta$ and the same set of parameters we used, the dependence on $\delta$ becomes significant.

${ }^{13}$ The helicity suppression of the dark matter annihilation into gluons plays an important role for this to happen.
} 


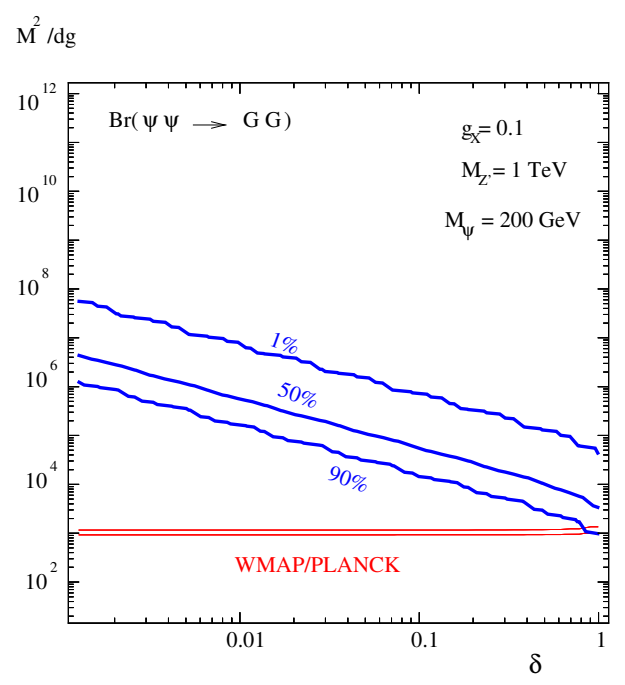

Figure 9. Gluonic branching fraction (blue line) of the annihilating dark matter in the plane $\left(\delta ; d_{g} / M^{2}\right)$ allowed by WMAP/PLANCK (red) data for a dark matter mass of $200 \mathrm{GeV}, g_{X}=0.1$ and $M_{Z^{\prime}}=1 \mathrm{TeV}$.

As explained earlier, parameters are allowed to lie below the red/full lines (Overdensity of the universe), above the orange/full line (LHC bounds on monojets production). Since the whole study has been released using effective dimension six operators generated by integrating out heavy fermions loops, one has to check that the parameter range is still in the window where $M \gg m_{\psi}$. This is indicated on figure 10 where we considered natural values of $d_{g}$ varying between $10^{-2}$ and 1 (purple and green/dashed line, respectively). Thus one can easily distinguish between the two regions $m_{\psi} \ll M$ (upper region) and $m_{\psi} \gg M$ (lower region).

In the case where $d_{g} \sim 10^{-2}$, it is important to notice that low values of the coupling constant $g_{X}$ provide almost no validity region in the parameter space since parameters have to lie above the purple/dashed line. On the other hand, for $g_{X}=1$ one can also notice that the allowed region is much larger in the case of a heavy $Z^{\prime}$. The case $d_{g} \sim 1$ considerably relax the constraints since the validity zones are almost in the region where $m_{\psi} \ll M$ (below the green/dashed line).

\section{$5 \quad Z^{\prime}$ annihilation into electroweak gauge bosons}

In the same way the $Z^{\prime}$ boson couples to gluons via operators of dimension six, mediators with electroweak quantum numbers can generate operators coupling the $Z^{\prime}$ boson to gauge bosons of the $\mathrm{SU}(2) \times \mathrm{U}(1)_{Y}$ electroweak sector. They can be parametrized as

$$
\begin{aligned}
\mathcal{L}=\frac{1}{M^{2}}\{ & D^{\mu} \theta_{X}\left[i\left(D^{\nu} H\right)^{\dagger}\left(c_{1} \tilde{F}_{\mu \nu}^{Y}+2 c_{2} \tilde{F}_{\mu \nu}^{W}\right) H+\text { h.c. }\right] \\
& +\partial^{m} D_{m} \theta_{X}\left(d_{1} \mathcal{T} r\left(F^{Y} \tilde{F}^{Y}\right)+2 d_{2} \mathcal{T} r\left(F^{W} \tilde{F}^{W}\right)\right)+d_{e w}^{\prime} \partial^{\mu} D^{\nu} \theta_{X} \operatorname{Tr}\left(F_{\mu \rho} \tilde{F}_{\nu}^{\rho}\right) \\
& \left.+e_{e w} D^{\mu} \theta_{X} \operatorname{Tr}\left(F_{\nu \rho} \mathcal{D}_{\mu} \tilde{F}^{\rho \nu}\right)+e_{e w}^{\prime} D_{\mu} \theta_{X} \operatorname{Tr}\left(F_{\alpha \nu} \mathcal{D}^{\nu} \tilde{F}^{\mu \alpha}\right)\right\} .
\end{aligned}
$$





Figure 10. Experimental constraints on $m_{\psi}$ and $M^{2} / d_{g}$ parameters, including LHC and universe overdensity constraints. Below the purple/dashed line $M \ll m_{\psi}$ and the effective theory analysis we made is not valid.

These effective operators give contributions to $Z^{\prime} \rightarrow Z Z, Z^{\prime} \rightarrow Z \gamma$ and $Z^{\prime} \rightarrow \gamma \gamma$ processes. We neglected such operators until now, since they induce new free parameters in the model. They can contribute to SM matter production in the universe, which in turn can slightly relax our previous constraints on the parameter $\frac{d_{g}}{M^{2}}$.

Let us now consider the $Z^{\prime}$ couplings to electroweak gauge bosons coming from the dimension-six operators $c_{i}$ and $d_{i}$ in (5.1), by ignoring the others. The reason for ignoring the last ones $d^{\prime}, e$ and $e^{\prime}$ is the same as for the gluonic couplings. On the other hand, although beyond the goals of the present paper, we believe that the operators $c_{i}$ are induced and do contribute in a computation with heavy loop of mediators, provided that part of mediator masses come from couplings to the SM Higgs. The interaction lagrangian of the couplings $c_{i}, d_{i}$ to the electroweak sector are then given by 
$\diamond Z^{\prime} \rightarrow Z Z$ process:

$$
\begin{aligned}
\Delta \mathcal{L}_{Z^{\prime} \rightarrow Z Z}= & g_{X} m_{Z} v \frac{\sin \theta_{W} c_{1}+\cos \theta_{W} c_{2}}{M^{2}} \epsilon_{\mu \nu \rho \sigma} Z^{\prime \mu} Z^{0 \nu} \partial^{\rho} Z^{0 \sigma} \\
& +2 \frac{\sin ^{2} \theta_{W} d_{1}+\cos ^{2} \theta_{W} d_{2}}{M^{2}} g_{X} \epsilon^{\mu \nu \rho \sigma} \partial^{m} Z_{m}^{\prime} \partial_{\mu} Z_{\nu} \partial_{\rho} Z_{\sigma},
\end{aligned}
$$

$\diamond Z^{\prime} \rightarrow Z \gamma$ process:

$$
\begin{aligned}
\Delta \mathcal{L}_{Z^{\prime} \rightarrow Z \gamma}= & g_{X} m_{Z} v \frac{\sin \theta_{W} c_{2}-\cos \theta_{W} c_{1}}{M^{2}} \epsilon_{\mu \nu \rho \sigma} Z^{\prime \mu} Z^{\nu 0} \partial^{\rho} A^{\sigma} \\
& +4 g_{X} \sin \theta_{W} \cos \theta_{W} \frac{d_{2}-d_{1}}{M^{2}} \epsilon^{\mu \nu \rho \sigma} \partial^{m} Z_{m}^{\prime} \partial_{\mu} Z_{\nu} \partial_{\rho} A_{\sigma}
\end{aligned}
$$

$\diamond Z^{\prime} \rightarrow W^{+} W^{-}$process:

$$
\begin{aligned}
\Delta \mathcal{L}_{Z^{\prime} \rightarrow W^{+} W^{-}}= & g_{X} v \frac{c_{2}}{M^{2}} Z^{\prime \mu} \epsilon_{\mu \nu \rho \sigma} m_{W}\left(W^{\nu-} \partial^{\rho} W^{+\sigma}+W^{\nu+} \partial^{\rho} W^{-\sigma}\right) \\
& +4 \frac{d_{2}}{M^{2}} g_{X} \epsilon^{\mu \nu \rho \sigma} \partial^{m} Z_{m}^{\prime} \partial_{\mu} W^{+}{ }_{\nu} \partial_{\rho} W^{-}{ }_{\sigma}
\end{aligned}
$$

$\diamond Z^{\prime} \rightarrow \gamma \gamma$ process:

$$
\Delta \mathcal{L}_{Z^{\prime} \rightarrow \gamma \gamma}=2 \frac{\cos ^{2} \theta_{W} d_{1}+\sin ^{2} \theta_{W} d_{2}}{M^{2}} g_{X} \epsilon^{\mu \nu \rho \sigma} \partial^{m} Z_{m}^{\prime} \partial_{\mu} A_{\nu} \partial_{\rho} A_{\sigma}
$$

These interaction terms give rise to the cross sections for the s-channel displayed in appendix D. They have to be added to the t-channel cross section. We can now add the resulting cross sections to the one of gluons production to consider a more precise constraint about universe overdensity, which is

$$
\left\langle\left(\sigma_{G G}+\sigma_{Z Z}+\sigma_{Z \gamma}+\sigma_{\gamma \gamma}+\sigma_{W^{+} W^{-}}\right) v\right\rangle_{\mathrm{s}-\text { channel }}+\langle\sigma v\rangle_{\mathrm{t}-\text { channel }} \geqslant\langle\sigma v\rangle_{\text {thermal }} .
$$

Then, assuming for simplicity that all the couplings appearing in the different sixdimensional operators are equal to $\frac{d_{g}}{M^{2}}$, which is a very strong hypothesis of course, we can plot a new constraint on this parameter, in a similar way we did before. This provides a new validity zone in the parameter space, as represented in figure 11 (in the case where $M_{Z^{\prime}}=1 \mathrm{TeV}$ and $g_{X}=1$ ), in which we added the electroweak processes to the gluon couplings of section 3 .

The resulting constraints are slightly relaxed, but the validity zones are not greatly enlarged, as anticipated earlier. One notice that the behaviour of the cross sections around $m_{\psi}=M_{Z^{\prime}} / 2$ is modified here, compared to the gluon production process. This happens because the electroweak gauge bosons $W^{ \pm}$and $Z$ are massive, unlike the gluons. Thus the Landau-Yang theorem does not apply and a real $Z^{\prime}$ can be created, relaxing the constraints on $M^{2} / d_{g}$ parameter. Implications of Landau-Yang theorem can yet be extended to express some constraints on what kind of CP even operators can be written down to produce electroweak gauge bosons; this has been done previously for $Z^{\prime} \rightarrow Z, Z$ process in [85]. Our results are in agreement with theirs in the form of operators and resulting cross sections. 


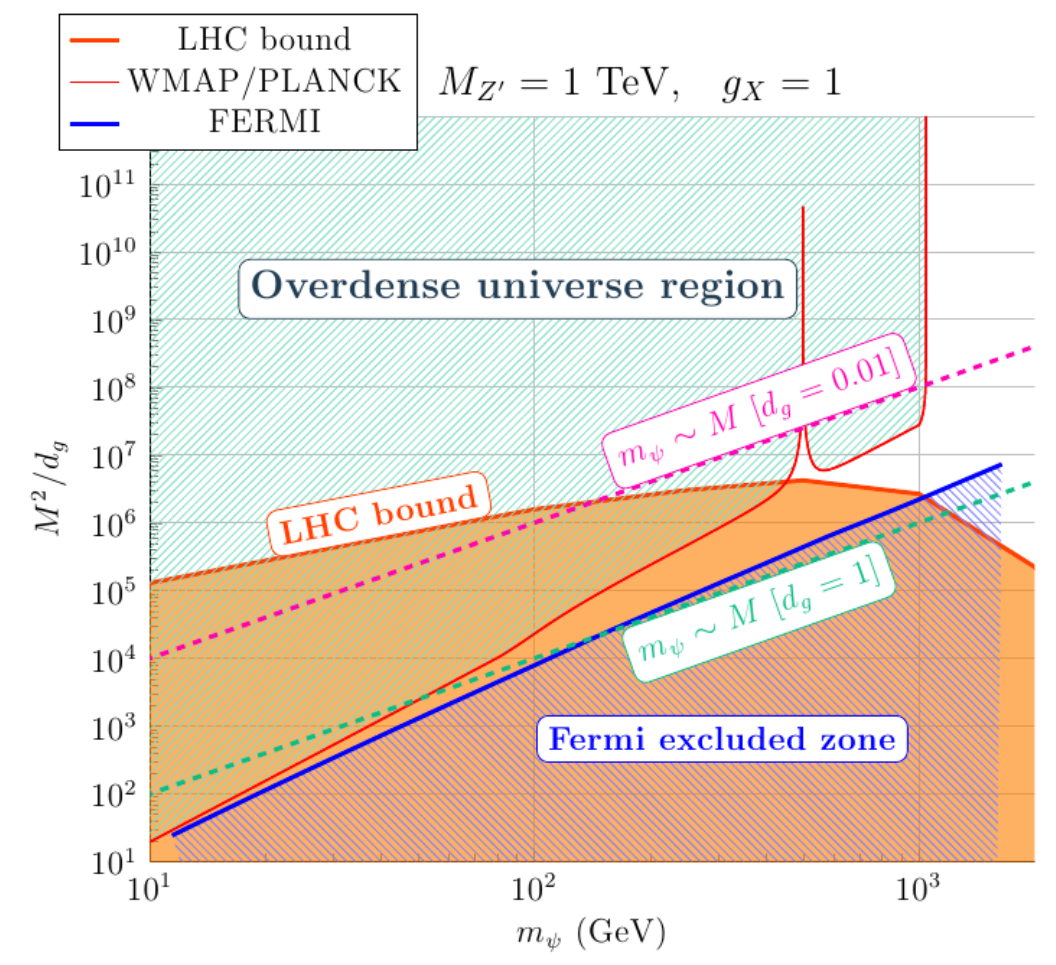

Figure 11. Experimental constraints on the $\left(M^{2} / d_{g}, m_{\psi}\right)$ parameters, taking into account dark matter couplings to all SM gauge bosons and assuming $c_{i}=d_{i}=d_{g}$.

\section{Acknowledgments}

We would like to thank Massimo Bianchi, J.M. Moreno and V. Martin-Lozano for very useful discussions. E.D., L.H and Y.M thank the Galileo Galilei Institute for Theoretical Physics for the hospitality and the INFN for partial support during the completion of this work. This work was supported in part by the European ERC Advanced Grant 226371 MassTeV, the French ANR TAPDMS ANR-09-JCJC-0146 the contract PITN-GA-2009237920 UNILHC and the Spanish MICINNs Consolider-Ingenio 2010 Programme under grant Multi- Dark CSD2009-00064. Y.M. acknowledges partial support from the European Union FP7 ITN INVISIBLES (Marie Curie Actions, PITN- GA-2011- 289442), the ERC advanced grant Higgs@LHC. B.Z. acknowledges the support of MICINN, Spain, under the contract FPA2010-17747, as well as the hospitality of LPT, Orsay, during the completion of this project.

\section{A Gauge independence and unitary gauge}

In this appendix we discuss the gauge independence of $Z^{\prime}$ induced effective couplings. In the Stueckelberg phase and after integrating out the heavy mediators, the effective action in $R_{\xi}$ gauges is

$$
\begin{aligned}
\mathcal{L}= & -\frac{1}{4}\left(F_{\mu \nu}^{Z^{\prime}}\right)^{2}+\frac{1}{2}\left(\partial_{\mu} a_{X}-\frac{g_{X}}{2} V Z_{\mu}^{\prime}\right)^{2}-\frac{1}{2 \xi}\left(\partial_{\mu} Z^{\prime \mu}+\xi \frac{g_{X}}{2} V a_{X}\right)^{2} \\
& +Z_{\mu}^{\prime} \Gamma^{\mu}(A)+a_{X} \Gamma_{a}(A)-m_{\psi}\left(e^{i a_{X}\left(X_{L}-X_{R}\right) / V} \bar{\psi}_{L} \psi_{R}+e^{-i a_{X}\left(X_{L}-X_{R}\right) / V} \bar{\psi}_{R} \psi_{L}\right) .
\end{aligned}
$$


In (A.1), $\Gamma^{\mu}(A)$ describes the local (non-local) coupling between $Z^{\prime}$ and SM gauge fields generated in the case where some heavy (light) fermions are charged under $Z^{\prime} . \Gamma_{a}$ is the axionic coupling generated in this case by the heavy set of mediator fermions cancelling an eventual gauge anomaly, which captures the low-energy remnant of the heavy mediator fermions in the infinite mass limit. Gauge invariance implies

$$
\partial_{\mu} \Gamma^{\mu}(A)=\frac{g_{X}}{2} V \Gamma_{a}(A) .
$$

At the abelian (three-point function) level, we can write

$$
\Gamma_{\mu}=\frac{1}{2} \Gamma_{\mu \nu \rho} A^{\nu} A^{\rho} \quad, \quad \Gamma^{a}=\frac{1}{2} \Gamma_{\nu \rho}^{a} A^{\nu} A^{\rho},
$$

where $A^{\nu}$ denotes symbolically the SM gauge fields. As concrete examples, the operator $\Gamma_{a}$ coupling gluons to the axion is of the form $\Gamma_{a} \sim \square \mathcal{T r}(G \tilde{G})+2 \partial_{\mu} \operatorname{Tr}\left(G_{\alpha \nu} D^{\nu} \tilde{G}^{\mu \alpha}\right)$ for the operators induced by chiral but anomaly-free set of heavy mediators in section 2.1 , whereas is of the form $\Gamma_{a} \sim \mathcal{T} r(G \tilde{G})$ for the anomalous sets of fermion mediators considered in section 2.2. In momentum space, the gauge invariance conditions for the three point function $Z^{\prime} A A$ are

$$
\begin{aligned}
& k_{1}^{\nu} \Gamma_{\mu \nu \rho}\left(k_{i}\right)=0 \quad, \quad k_{2}^{\rho} \Gamma_{\mu \nu \rho}\left(k_{i}\right)=0 \\
& i\left(k_{1}+k_{2}\right)^{\mu} \Gamma_{\mu \nu \rho}\left(k_{i}\right)=\frac{g_{X}}{2} V \Gamma_{\nu \rho}^{a}\left(k_{i}\right) .
\end{aligned}
$$

The $Z^{\prime}$ and axion propagators are

$$
\Delta_{\mu \nu}^{Z^{\prime}}(q)=-i \frac{\eta_{\mu \nu}+(\xi-1) \frac{q_{\mu} q_{\nu}}{q^{2}-\xi M_{Z^{\prime}}^{2}}}{q^{2}-M_{Z^{\prime}}^{2}} \quad, \quad \Delta_{a_{X}}(q)=\frac{i}{q^{2}-\xi M_{Z^{\prime}}^{2}}
$$

and the unitary gauge corresponds to the limit $\xi \rightarrow \infty$. Whereas the issue of gauge-fixing independence can be discussed in more general terms, we prefer to analyse it in the relevant context for our work, fermions- 2 SM gauge fields interactions mediated by the $Z^{\prime}$ exchange. In an arbitrary $R_{\xi}$ gauge, there are two contributions: the $Z^{\prime}$ and the axionic exchange:

$$
\begin{aligned}
& \mathcal{M}=\bar{v}\left(p_{2}\right)\left(-\frac{i g_{X}}{2}\right)\left[\frac{X_{R}+X_{L}}{2} \gamma^{\mu}+\frac{X_{R}-X_{L}}{2} \gamma^{\mu} \gamma_{5}\right] u\left(p_{1}\right)\left(-i \frac{\eta_{\mu \nu}+(\xi-1) \frac{q_{\mu} q_{\nu}}{q^{2}-\xi M_{Z^{\prime}}^{2}}}{q^{2}-M_{Z^{\prime}}^{2}}\right) \Gamma^{\nu} \\
& +\bar{v} \gamma_{5}\left(X_{L}-X_{R}\right) \frac{m_{\psi}}{V} \frac{i}{q^{2}-\xi M_{Z^{\prime}}^{2}} \Gamma_{a} u\left(p_{1}\right),
\end{aligned}
$$

where $\Gamma^{\nu}, \Gamma_{a}$ are the three-point functions coming from the operators present in (A.1), $q$ is the $Z^{\prime}$ virtual momentum and $u(p), v(p)$ the Dirac spinors associated to the fermion (antifermion) $\Psi$ coupling to $Z^{\prime}$, to be identified with the Dark Matter candidate in our paper. By using Dirac equation for the fermion $\Psi$ and the gauge invariance condition (A.2) in momentum space $-i q_{\mu} \Gamma^{\mu}\left(k_{i}\right)=\frac{g_{X}}{2} V \Gamma_{a}\left(k_{i}\right)$, with $k_{1}, k_{2}$ the momenta of the two gauge bosons in the final space, we find

$$
\begin{aligned}
& \mathcal{M}=\bar{v}\left(p_{2}\right)\left(-\frac{i g_{X}}{2}\right)\left[\frac{X_{R}+X_{L}}{2} \gamma^{\mu}+\frac{X_{R}-X_{L}}{2} \gamma^{\mu} \gamma_{5}\right] u\left(p_{1}\right)\left(\frac{-i \Gamma^{\mu}}{q^{2}-M_{Z^{\prime}}^{2}}\right) \\
& +\bar{v}\left(p_{2}\right) \gamma_{5}\left(X_{L}-X_{R}\right) \frac{m_{\psi}}{V} \frac{i}{q^{2}-M_{Z^{\prime}}^{2}} \Gamma_{a} u\left(p_{1}\right) .
\end{aligned}
$$


As expected, due to gauge invariance, the $\xi$-dependence cancelled in the final result. Moreover, the result can also be directly found in the unitary gauge with no axion field present. In this case, the result is fully encoded in the unitary gauge computation

$$
\mathcal{M}=\bar{v}\left(p_{2}\right)\left(-\frac{i g_{X}}{2}\right)\left[\frac{X_{R}+X_{L}}{2} \gamma^{\mu}+\frac{X_{R}-X_{L}}{2} \gamma^{\mu} \gamma_{5}\right] u\left(p_{1}\right)\left(-i \frac{\eta_{\mu \nu}-\frac{q_{\mu} q_{\nu}}{M_{Z^{\prime}}^{2}}}{q^{2}-M_{Z^{\prime}}^{2}}\right) \Gamma^{\nu}
$$

Notice that in the unitary gauge the lagrangian can be expressed entirely in terms of

$$
\tilde{Z}_{\mu}^{\prime}=Z_{\mu}^{\prime}-\frac{2}{g_{X} V} \partial_{\mu} a_{X} \quad, \quad \tilde{\Psi}_{L, R}=e^{-\frac{i a_{X}}{V} X_{L, R}} \Psi_{L, R} .
$$

Unitary gauge captures correctly the result in the infinite mass limit of the heavy fermions. For finite masses, there are corrections which are not captured by the naive unitary gauge computation.

\section{B Three-point gauge boson amplitude and gauge effective action from heavy fermion loops: couplings to gluons}

In the case of $\mathrm{CP}$ invariance, the three-point gauge boson amplitude can be generally be written as $[10-12]$

$$
\begin{aligned}
\Gamma^{\mu \nu \rho}= & \epsilon^{\mu \nu \rho \alpha}\left(A_{1} k_{1 \alpha}+A_{2} k_{2 \alpha}\right) \\
& +\left[\epsilon^{\mu \nu \alpha \beta}\left(B_{1} k_{1}^{\rho}+B_{2} k_{2}^{\rho}\right)+\epsilon^{\mu \rho \alpha \beta}\left(B_{3} k_{1}^{\nu}+B_{4} k_{2}^{\nu}\right)\right] k_{1 \alpha} k_{2 \beta},
\end{aligned}
$$

where $A_{i}, B_{i}$ are Lorentz-invariant functions of the external momenta $k_{i}$. The functions $A_{i}$ which encode the generalized Chern-Simon terms (GCS) [10-12] are superficially logarithmically divergent, whereas the functions $B_{i}$ are UV finite. However, $A_{i}$ are determined in terms of $B_{i}$ by using the Ward identities, which in case the heavy fermions form an anomaly-free set, are given by

$$
\begin{aligned}
& k_{1}^{\nu} \Gamma_{\mu \nu \rho}=0 \rightarrow \quad A_{2}=B_{3} k_{1}^{2}+B_{4} k_{1} k_{2}, \\
& k_{2}^{\rho} \Gamma_{\mu \nu \rho}=0 \quad \rightarrow \quad A_{1}=B_{2} k_{2}^{2}+B_{1} k_{1} k_{2}, \\
&-\left(k_{1}+k_{2}\right)^{\mu} \Gamma_{\mu \nu \rho}=\left(A_{1}-A_{2}\right) \epsilon_{\nu \rho \alpha \beta} k_{1}^{\alpha} k_{2}^{\beta} .
\end{aligned}
$$

The last current conservation is nontrivial in our case, since gauge invariance is realized through an additional axionic coupling to gauge fields generated by heavy fermions, such that we find (A.4). After comparison with (B.2), this implies

$$
\Gamma_{\nu \rho}^{a}=-\frac{2 i}{g_{X} V}\left(A_{1}-A_{2}\right) \epsilon_{\nu \rho \alpha \beta} k_{1}^{\alpha} k_{2}^{\beta} .
$$

The situation here is different compared to the usual discussion of anomalies. The usual axionic couplings compensating triangle gauge anomalies are generated by chiral and nonanomaly free set of fermions. If the heavy fermions form an anomaly-free set, they do not generate such couplings, but dimension six operators for gauge fields and dimension seven 
axionic couplings, which cancel between themselves their gauge variation. At the one loop order, there are two contributions to $\Gamma^{\mu \nu \rho}$. The first is the triangle loop diagram with no chirality flip/mass insertions, given by

$$
\Gamma_{\mu \nu \rho}^{(1)}=\sum_{i} t_{i a a} \int \frac{d^{4} p}{(2 \pi)^{4}} \operatorname{Tr}\left[\frac{\not p+\not k_{2}}{\left(p+k_{2}\right)^{2}-M_{i}^{2}} \gamma_{\rho} \frac{\not p}{p^{2}-M_{i}^{2}} \gamma_{\nu} \frac{\not p-\not k_{1}}{\left(p-k_{1}\right)^{2}-M_{i}^{2}} \gamma_{\mu} \gamma_{5}\right] .
$$

where $t_{i a a}=\operatorname{Tr}\left(X_{i} T^{a} T^{a}\right)$. There are also three other contributions with two mass insertions, of the type

$$
\Gamma_{\mu \nu \rho}^{(2)}=\sum_{i} t_{i a a} \int \frac{d^{4} p}{(2 \pi)^{4}} \operatorname{Tr}\left[\frac{M_{i}}{\left(p+k_{2}\right)^{2}-M_{i}^{2}} \gamma_{\rho} \frac{\not p}{p^{2}-M_{i}^{2}} \gamma_{\nu} \frac{M_{i}}{\left(p-k_{1}\right)^{2}-M_{i}^{2}} \gamma_{\mu} \gamma_{5}\right]+\cdots,
$$

where $\cdots$ denote two similar contributions with the mass insertions permuted among the three propagators. By using a Feynman parametrization and after performing a shift of the momentum integral $p \rightarrow p+\beta k_{1}-\alpha k_{2}$, we find

$$
\begin{aligned}
\Gamma_{\mu \nu \rho}^{(1)}=2 \sum_{i} t_{i a a, L} & -R \int_{0}^{1} d \alpha \int_{0}^{1} d \beta \times \\
& \times \int \frac{d^{4} p}{(2 \pi)^{4}} \frac{N_{\mu \nu \rho}\left(p, k_{i}\right)}{\left[p^{2}+\alpha(1-\alpha) k_{2}^{2}+\beta(1-\beta) k_{1}^{2}+2 \alpha \beta k_{1} k_{2}-M_{i}^{2}\right]^{3}},
\end{aligned}
$$

where $t_{i a a, L-R}=\operatorname{Tr}\left[\left(X_{L}-X_{R}\right) T_{a} T_{a}\right]_{i}$ and where

$$
\begin{aligned}
& \left.N_{\mu \nu \rho}\left(p, k_{i}\right)=\operatorname{Tr}\left\{\not \not \phi+\beta \not k_{1}+(1-\alpha) \not k_{2}\right] \gamma_{\rho}\left[\not \phi+\beta \not k_{1}-\alpha \not k_{2}\right] \gamma_{\nu}\left[\not \phi-(1-\beta) \not k_{1}-\alpha \not k_{2}\right] \gamma_{\mu} \gamma_{5}\right\} \\
& =-\operatorname{Tr}\left\{\not p \gamma_{\rho} \not\left[\left[(1-\beta) \not \phi_{1}+\alpha \not k_{2}\right] \gamma_{\mu} \gamma_{5}\right\}+\operatorname{Tr}\left\{\left[\beta \not k_{1}+(1-\alpha) \not k_{2}\right] \gamma_{\rho} \not \rho \gamma_{\nu} \not \gamma \gamma_{\mu} \gamma_{5}\right\}\right. \\
& +\operatorname{Tr}\left\{\not p \gamma_{\rho}\left[\beta \not \phi_{1}-\alpha \not \phi_{2}\right] \gamma_{\nu} \not \gamma \gamma_{\mu} \gamma_{5}\right\} \\
& -\operatorname{Tr}\left\{\left[\beta \not k_{1}+(1-\alpha) \not k_{2}\right] \gamma_{\rho}\left[\beta \not k_{1}-\alpha \not k_{2}\right] \gamma_{\nu}\left[(1-\beta) \not \not_{1}+\alpha \not k_{2}\right] \gamma_{\mu} \gamma_{5}\right\}
\end{aligned}
$$

The first three terms in (B.7) contribute to the ambiguous $A_{i}$ functions which will be however uniquely determined by the Ward identities (B.2). The last one, on the other hand, is contributing to $B_{i}$ and equals

$$
\begin{aligned}
\operatorname{Tr}\left\{\left[\beta \not k_{1}\right.\right. & \left.\left.+(1-\alpha) \not k_{2}\right] \gamma_{\rho}\left[\beta \not k_{1}-\alpha \not k_{2}\right] \gamma_{\nu}\left[(1-\beta) \not k_{1}+\alpha \not k_{2}\right] \gamma_{\mu} \gamma_{5}\right\} \\
=- & 4 i\left\{\left[\beta(2 \alpha+\beta-1) k_{1 \rho}+\alpha(2-2 \alpha-\beta) k_{2 \rho}\right] \epsilon_{\mu \nu \alpha \beta} k_{1}^{\alpha} k_{2}^{\beta}\right. \\
& -\beta\left[(1-\beta) k_{1 \mu}+\alpha k_{2 \mu}\right] \epsilon_{\nu \rho \alpha \beta} k_{1}^{\alpha} k_{2}^{\beta}+\beta\left[(1-\beta) k_{1 \mu}+\alpha k_{2 \mu}\right] \epsilon_{\rho \mu \alpha \beta} k_{1}^{\alpha} k_{2}^{\beta} \\
& \left.-\epsilon_{\mu \nu \rho \alpha}\left[\beta^{2} k_{1}^{2}-\alpha(1-\alpha) k_{2}^{2}+(1-2 \alpha) \beta k_{1} k_{2}\right]\left[(1-\beta) k_{1}^{\alpha}+\alpha k_{2}^{\alpha}\right]\right\}
\end{aligned}
$$

Integrating over the internal momentum $p$ and over the Feynman parameters $\alpha, \beta$ one finally finds

$$
\begin{aligned}
\Gamma_{\mu \nu \rho}^{(1)}= & -\sum_{i} \frac{i t_{i a a, L-R}}{48 \pi^{2} M_{i}^{2}}\left\{\left(4 k_{1}+k_{2}\right)_{\rho} \epsilon_{\mu \nu \alpha \beta}-\left(2 k_{1}+3 k_{2}\right)_{\mu} \epsilon_{\nu \rho \alpha \beta}+\left(2 k_{1}+3 k_{2}\right)_{\nu} \epsilon_{\rho \mu \alpha \beta}\right\} k_{1}^{\alpha} k_{2}^{\beta} \\
& +\mathrm{A}-\text { terms }
\end{aligned}
$$


where the A terms in (B.1) are determined at the end by the Ward identity (B.2). The last step is the symmetrization in the two gluonic legs, which leads to the final result

$$
\begin{aligned}
\Gamma_{\mu \nu \rho}^{(1) \text { symm. }}=- & -\sum_{i} \frac{i t_{i a a, L-R}}{48 \pi^{2} M_{i}^{2}}\left\{\left(7 k_{1}+3 k_{2}\right)_{\rho} \epsilon_{\mu \nu \alpha \beta}-5\left(k_{1}+k_{2}\right)_{\mu} \epsilon_{\nu \rho \alpha \beta}\right. \\
& \left.\left.+\left(3 k_{1}+7 k_{2}\right)_{\nu} \epsilon_{\rho \mu \alpha \beta}\right\} k_{1}^{\alpha} k_{2}^{\beta}+\cdots \quad \text { (B. } 10\right) \\
= & -\sum_{i} \frac{i t_{i a a, L-R}}{12 \pi^{2} M_{i}^{2}}\left\{\left(-k_{1 \rho} \epsilon_{\mu \nu \alpha \beta}+2\left(k_{1}+k_{2}\right)_{\mu} \epsilon_{\nu \rho \alpha \beta}-k_{2 \nu} \epsilon_{\rho \mu \alpha \beta}\right\} k_{1}^{\alpha} k_{2}^{\beta}+\mathrm{A}-\text { terms },\right.
\end{aligned}
$$

where in order to find the last line we used the identities

$$
\begin{aligned}
& \left(\epsilon^{\nu \rho \alpha \beta} k_{1}^{\mu}+\epsilon^{\rho \mu \alpha \beta} k_{1}^{\nu}+\epsilon^{\mu \nu \alpha \beta} k_{1}^{\rho}\right) k_{1 \alpha} k_{2 \beta}=\epsilon^{\mu \nu \rho \alpha}\left(k_{1}^{2} k_{2 \alpha}-k_{1} k_{2} k_{1 \alpha}\right), \\
& \left(\epsilon^{\nu \rho \alpha \beta} k_{2}^{\mu}+\epsilon^{\rho \mu \alpha \beta} k_{2}^{\nu}+\epsilon^{\mu \nu \alpha \beta} k_{2}^{\rho}\right) k_{1 \alpha} k_{2 \beta}=\epsilon^{\mu \nu \rho \alpha}\left(k_{1} k_{2} k_{2 \alpha}-k_{2}^{2} k_{1 \alpha}\right) .
\end{aligned}
$$

The contribution with two mass insertions $\Gamma_{\mu \nu \rho}^{(2)}$ are easily seen to give terms correcting the coefficients $A_{i}$ in (B.1). As such, they are fixed by the Ward identities (B.2). At the one loop order, the complete three-point function, including the $A_{i}$ coefficients defined in (B.1), is then given by

$$
\Gamma_{\mu \nu \rho}^{\mathcal{O}}=-\sum_{i} \frac{i t_{i a a, L-R}}{12 \pi^{2} M_{i}^{2}}\left\{\left[2\left(k_{1}+k_{2}\right)_{\mu} \epsilon_{\nu \rho \alpha \beta}-k_{1 \rho} \epsilon_{\mu \nu \alpha \beta}-k_{2 \nu} \epsilon_{\rho \mu \alpha \beta}\right] k_{1}^{\alpha} k_{2}^{\beta}+\epsilon_{\mu \nu \rho \alpha} k_{1} k_{2}\left(k_{2}-k_{1}\right)^{\alpha}\right\} .
$$

Notice that (B.12) can be cast in the general form (B.1). Indeed, by using identities of the type (B.11), one can also write

$$
\begin{array}{r}
\Gamma_{\mu \nu \rho}^{\mathcal{O}}=\sum_{i} \frac{i t_{i a a, L-R}}{12 \pi^{2} M_{i}^{2}}\left\{\left[\left(3 k_{1 \rho}+2 k_{2 \rho}\right) \epsilon_{\mu \nu \alpha \beta}+\left(2 k_{1 \nu}+3 k_{2 \nu}\right) \epsilon_{\rho \mu \alpha \beta}\right] k_{1}^{\alpha} k_{2}^{\beta}\right. \\
\left.+\epsilon_{\mu \nu \rho \alpha}\left[\left(2 k_{1}^{2}+3 k_{1} k_{2}\right) k_{2}^{\alpha}-\left(2 k_{2}^{2}+3 k_{1} k_{2}\right) k_{1}^{\alpha}\right]\right\},
\end{array}
$$

from which the coeff. $A_{i}, B_{i}$ in (B.1) can be readily identified. The final result for the $Z^{\prime}$ couplings is then described by the operator

$$
\mathcal{O}=\frac{g_{3}^{2}}{24 \pi^{2}} \sum_{i} \operatorname{Tr}\left(\frac{\left(X_{L}-X_{R}\right) T_{a} T_{a}}{M^{2}}\right)_{i}\left[\partial^{\mu} D_{\mu} \theta_{X} \mathcal{T} r(G \tilde{G})-2 D_{\mu} \theta_{X} \operatorname{Tr}\left(G_{\alpha \nu} \mathcal{D}^{\nu} \tilde{G}^{\mu \alpha}\right)\right]
$$

The antisymmetric part of (B.9), which is relevant if one replaces $Z^{\prime}$ by another gluon, can be shown to vanish, by using the identities (B.11). Therefore, one-loops of heavy mediators do not generate triple SM gauge boson vectors operators of the type (2.16) and there are no new phenomenological constraints coming from purely SM contact operators.

\section{Vanishing of the operator $\mathcal{T} r\left(F^{X} F_{\mathrm{SM}} \tilde{F}_{\mathrm{SM}}\right)$ and a useful identity}

Here we show that the operator $\operatorname{Tr}\left(F^{X} F_{\mathrm{SM}} \tilde{F}_{\mathrm{SM}}\right)$ is identically zero. The proof is the same for any SM gauge field, so we consider the gluons for definiteness. In the unitary gauge, the $Z^{\prime}$-gluon-gluon vertex coming from this operator is proportional to

$$
\frac{1}{M^{2}} \epsilon^{\lambda \nu \rho \sigma}\left(\partial_{\mu} Z_{\nu}^{\prime} \partial^{\mu} G_{\lambda}^{A} \partial_{\rho} G_{\sigma}^{A}-\partial_{\mu} Z_{\nu}^{\prime} \partial_{\lambda} G_{\mu}^{A} \partial_{\rho} G_{\sigma}^{A}-\partial_{\nu} Z_{\mu}^{\prime} \partial^{\mu} G_{\lambda}^{A} \partial_{\rho} G_{\sigma}^{A}+\partial_{\nu} Z_{\mu}^{\prime} \partial_{\lambda} G_{\mu}^{A} \partial_{\rho} G_{\sigma}^{A}\right)
$$


In momentum space, denoting by $k_{1}, k_{2}$ the momenta of the two gluons, the linearized (abelian) $Z^{\prime} G G$ vertex, after symmetrization of the two gluons, is given by

$$
\Gamma^{\mu \nu \rho}=\epsilon^{\nu \rho \sigma \tau} k_{1 \tau} k_{2}^{\mu} k_{2}^{\sigma}+\epsilon^{\nu \rho \mu \sigma}\left(k_{1} k_{2} k_{1 \sigma}-k_{1}^{2} k_{2 \sigma}\right)+\epsilon^{\rho \mu \sigma \tau} k_{1} \nu k_{1 \sigma} k_{2}^{\tau}-\epsilon^{\nu \mu \sigma \tau} k_{1} \rho k_{1 \sigma} k_{2}^{\tau} .
$$

Its vanishing can be seen by starting from the identity

$$
\left(\epsilon^{\nu \rho \sigma \tau} k_{3}^{\mu}+\epsilon^{\rho \mu \sigma \tau} k_{3}^{\nu}+\epsilon^{\mu \nu \sigma \tau} k_{3}^{\rho}\right) k_{2}^{\sigma} k_{1}^{\tau}=\epsilon^{\mu \nu \rho \tau}\left(k_{2} k_{3} k_{1 \tau}-k_{1} k_{3} k_{2 \tau}\right)
$$

The identity is actually valid for any vector $k_{3}$, that can be chosen, as in (B.11), to be one of the gluon momenta $k_{1,2}$, or the $Z^{\prime}$ momentum $k_{3}=-\left(k_{1}+k_{2}\right)$.

If the linearized abelian part of the operator vanishes, it has to completely vanish because of gauge invariance.

\section{The s and t-channel dark matter annihilation cross sections}

\section{D.1 The s-channel electroweak annihilation cross sections into electroweak gauge bosons}

The interaction terms of coeff. $c_{i}, d_{i}$ in (5.1) give rise to the following cross sections for the s-channel

$\diamond Z^{\prime} \rightarrow Z Z$ process:

$$
\begin{aligned}
& \sigma_{\psi^{\mathrm{DM}}, \psi^{\mathrm{DM}} \rightarrow Z, Z} \\
& =\left(\frac{\sin \theta_{W} c_{1}+\cos \theta_{W} c_{2}}{M^{2}}\right)^{2} \frac{v^{2} g_{X}^{4}\left(s-4 m_{Z}^{2}\right)}{\left(M_{Z^{\prime}}^{2}-s\right)^{2}+M_{Z^{\prime}}^{2} \Gamma\left(Z^{\prime}\right)^{2}} \sqrt{\frac{s-4 m_{Z}^{2}}{s-4 m_{\psi}^{2}}} \times \\
& \quad \times \frac{M_{Z^{\prime}}^{4}\left(s-4 m_{Z}^{2}\right)\left(X_{L}^{2}+X_{R}^{2}\right)\left(2 m_{\psi}^{2}+s\right)+m_{\psi}^{2}\left(X_{L}-X_{R}\right)^{2}\left(6 m_{Z}^{2}\left(s-M_{Z^{\prime}}^{2}\right)^{2}-3 M_{Z^{\prime}}^{4}\left(s-4 m_{Z}^{2}\right)\right)}{768 \pi M_{Z^{\prime}}^{4} s},
\end{aligned}
$$

$\diamond Z^{\prime} \rightarrow \gamma Z$ process:

$$
\begin{aligned}
& \sigma_{\psi^{\mathrm{DM}}}^{\mathrm{DMM}} \rightarrow \gamma Z \\
&=\frac{\theta\left(s-m_{Z}^{2}\right) g_{X}^{4}}{\Gamma\left(Z^{\prime}\right)^{2} M_{Z^{\prime}}^{2}+\left(M_{Z^{\prime}}^{2}-s\right)^{2}} \sqrt{\frac{s}{s-4 m_{\psi}^{2}}} \\
& \quad \times\left(\sin ^{2} \theta_{W} \cos ^{2} \theta_{W} \frac{\left(d_{2}-d_{1}\right)^{2}}{M^{4}} \frac{m_{\psi}^{2}\left(X_{L}-X_{R}\right)^{2}\left(s-m_{Z}^{2}\right)^{3}\left(s-M_{Z^{\prime}}^{2}\right)^{2}}{4 \pi M_{Z^{\prime}}^{4}}+v^{2} \frac{\left(\sin \theta_{W} c_{2}-\cos \theta_{W} c_{1}\right)^{2}}{M^{4}} \times\right. \\
& \quad \times \frac{\left(m_{Z}^{2}-s\right)^{3}\left(M_{Z^{\prime}}^{4}\left(2 m_{\psi}^{2}+s\right)\left(m_{Z}^{2}+s\right)\left(X_{L}^{2}+X_{R}^{2}\right)+m_{\psi}^{2}\left(X_{L}-X_{R}\right)^{2}\left(-6 m_{Z}^{2} M_{Z^{\prime}}^{2} s+3 m_{Z}^{2} s^{2}-3 M_{Z^{\prime}}^{4} s\right)\right)}{768 \pi M_{Z^{\prime}}^{4} s^{3}} \\
&\left.-m_{Z} v \frac{\left(\sin \theta_{W} c_{2}-\cos \theta_{W} c_{1}\right)\left(d_{2}-d_{1}\right)}{M^{4}} \times \frac{m_{\psi}^{2}\left(X_{L}-X_{R}\right)^{2}\left(s-m_{Z}^{2}\right)^{3}\left(s-M_{Z^{\prime}}^{2}\right)^{2}}{8 \pi M_{Z^{\prime}}^{4}}\right),
\end{aligned}
$$


$\diamond Z^{\prime} \rightarrow \gamma \gamma$ process:

$$
\begin{aligned}
\sigma_{\psi^{\mathrm{DM}} \psi^{\mathrm{DM}} \rightarrow \gamma \gamma}= & \frac{\left(\cos ^{2} \theta_{W} d_{1}+\sin ^{2} \theta_{W} d_{2}\right)^{2}}{M^{4}} \frac{\left(-s+M_{Z^{\prime}}^{2}\right)^{2}}{\left(-s+M_{Z^{\prime}}^{2}\right)^{2}+M_{Z^{\prime}}^{2} \Gamma\left(Z^{\prime}\right)^{2}} \times \\
& \times \frac{g_{X}^{4} m_{\psi}^{2} s^{2}\left(X_{L}-X_{R}\right)^{2}}{32 \pi M_{Z^{\prime}}^{4}} \sqrt{\frac{s}{s-4 m_{\psi}^{2}}} .
\end{aligned}
$$

Notice the vanishing of the cross-section for the on-shell $Z^{\prime}$ case $s=M_{Z^{\prime}}^{2}$, in agreement with the Landau-Yang theorem [83, 84].

$\diamond Z^{\prime} \rightarrow W^{+} W^{-}$process:

$$
\begin{aligned}
& \sigma_{\psi^{\mathrm{DM}}}^{\mathrm{D}} \psi^{\mathrm{DM}} \rightarrow W^{+} W^{-} \\
&= \frac{\theta\left(s-4 m_{W}^{2}\right)\left(s-4 m_{W}^{2}\right)^{3 / 2} g_{X}^{4}}{\left(\Gamma^{2} M_{Z^{\prime}}^{2}+\left(M_{Z^{\prime}}^{2}-s\right)^{2}\right) \sqrt{s-4 m_{\psi}^{2}}} \\
& \times\left(\left(\frac{d_{2}}{M^{2}}\right)^{2} \frac{m_{\psi}^{2} s\left(M_{Z^{\prime}}^{2}-s\right)^{2}\left(X_{L}-X_{R}\right)^{2}}{16 \pi M_{Z^{\prime}}^{4}}+\left(\frac{c_{2}}{M^{2}}\right)\left(\frac{d_{2}}{M^{2}}\right) \frac{v m_{W}\left(M_{Z^{\prime}}^{2}-s\right)^{2}\left(m_{\psi}^{2}\left(X_{L}-X_{R}\right)^{2}\right)}{16 \pi M_{Z^{\prime}}^{4}}\right. \\
&\left.+\left(\frac{c_{2} v}{M^{2}}\right)^{2}\left(\frac{\left(s-4 m_{W}^{2}\right)\left(X_{L}^{2}+X_{R}^{2}\right)\left(2 m_{\psi}^{2}+s\right)}{384 \pi s}+\frac{m_{\psi}^{2}\left(X_{L}-X_{R}\right)^{2}\left(6 m_{W}^{2}\left(s-M_{Z^{\prime}}^{2}\right)^{2}-3 M_{Z^{\prime}}^{4}\left(s-4 m_{W}^{2}\right)\right)}{384 \pi M_{Z^{\prime}}^{4} s}\right)\right) .
\end{aligned}
$$

\section{D.2 The t-channel dark matter annihilation into $Z^{\prime} Z^{\prime}$}

We give here the exact formula of the t-channel process cross-section as a function of the center of mass energy squared $s$ :

$$
\begin{gathered}
\langle\sigma v\rangle_{\mathrm{t}-\mathrm{ch} .}=\frac{g_{X}^{4} v}{1024 \pi^{2} M_{Z^{\prime}}^{4} s} \sqrt{\frac{s-4 M_{Z^{\prime}}^{2}}{s-4 m_{\psi}^{2}}}\left\{-2 m_{\psi}^{2}\left(4 M_{Z^{\prime}}^{2}-s\right)\left(X_{L}-X_{R}\right)^{4}-8 M_{Z^{\prime}}^{4}\left(X_{L}^{4}+X_{R}^{4}\right)\right. \\
+\frac{1}{\left(2 M_{Z^{\prime}}^{2}-s\right) \sqrt{\left(s-4 m_{\psi}^{2}\right)\left(s-4 M_{Z^{\prime}}^{2}\right)}} 8 \operatorname{coth}^{-1}\left(\frac{2 M_{Z^{\prime}}^{2}-s}{\sqrt{\left(s-4 m_{\psi}^{2}\right)\left(s-4 M_{Z^{\prime}}^{2}\right)}}\right) \\
\quad \times\left[m _ { \psi } ^ { 4 } \left(2 M_{Z^{\prime}}^{4}\left(3 X_{L}-X_{R}\right)\left(X_{L}+X_{R}\right)^{2}\left(X_{L}-3 X_{R}\right)+4 M_{Z^{\prime}}^{2} s\left(X_{L}-X_{R}\right)^{4}\right.\right. \\
\left.-s^{2}\left(X_{L}-X_{R}\right)^{4}\right)+2 m_{\psi}^{2} M_{Z^{\prime}}^{2}\left(4 M _ { Z ^ { \prime } } ^ { 4 } \left(-2 X_{L}^{4}+X_{L}^{3} X_{R}-2 X_{L}^{2} X_{R}^{2}\right.\right. \\
\left.\quad+X_{L} X_{R}^{3}-2 X_{R}^{4}\right)+s^{2}\left(X_{L}-X_{R}\right)^{2}\left(X_{L}^{2}+X_{R}^{2}\right) \\
\left.+2 M_{Z^{\prime}}^{2} s\left(-3 X_{L}^{4}+4 X_{L}^{3} X_{R}+2 X_{L}^{2} X_{R}^{2}+4 X_{L} X_{R}^{3}-3 X_{R}^{4}\right)\right) \\
\left.+2 M_{Z^{\prime}}^{4}\left(4 M_{Z^{\prime}}^{4}+s^{2}\right)\left(X_{L}^{4}+X_{R}^{4}\right)\right] \\
4 M_{Z^{\prime}}^{4} \\
+\frac{m_{\psi}^{2}(s-}{\left.4 M_{Z^{\prime}}^{2}\right)+M_{Z^{\prime}}^{4}}\left[m_{\psi}^{4}\left(X_{L}^{2}-6 X_{L} X_{R}+X_{R}^{2}\right)^{2}\right. \\
+2 m_{\psi}^{2}\left(M_{Z^{\prime}}^{2}\left(-3 X_{L}^{4}+6 X_{L}^{3} X_{R}+2 X_{L}^{2} X_{R}^{2}+6 X_{L} X_{R}^{3}-3 X_{R}^{4}\right)\right. \\
\left.\left.\left.+s\left(X_{L}^{2}-X_{R}^{2}\right)^{2}\right)+2 M_{Z^{\prime}}^{4}\left(X_{L}^{4}+X_{R}^{4}\right)\right]\right\}
\end{gathered}
$$

Open Access. This article is distributed under the terms of the Creative Commons Attribution License which permits any use, distribution and reproduction in any medium, provided the original author(s) and source are credited. 


\section{References}

[1] P. Langacker, The physics of heavy $Z^{\prime}$ gauge bosons, Rev. Mod. Phys. 81 (2009) 1199 [arXiv:0801.1345] [INSPIRE].

[2] T.G. Rizzo, $Z^{\prime}$ phenomenology and the $L H C$, hep-ph/0610104 [INSPIRE].

[3] M.S. Carena, A. Daleo, B.A. Dobrescu and T.M. Tait, $Z^{\prime}$ gauge bosons at the Tevatron, Phys. Rev. D 70 (2004) 093009 [hep-ph/0408098] [InSPIRE].

[4] E. Salvioni, G. Villadoro and F. Zwirner, Minimal $Z^{\prime}$ models: present bounds and early LHC reach, JHEP 11 (2009) 068 [arXiv:0909.1320] [INSPIRE].

[5] E. Salvioni, A. Strumia, G. Villadoro and F. Zwirner, Non-universal minimal $Z^{\prime}$ models: present bounds and early LHC reach, JHEP 03 (2010) 010 [arXiv:0911.1450] [INSPIRE].

[6] M. Cvetič and P. Langacker, Implications of abelian extended gauge structures from string models, Phys. Rev. D 54 (1996) 3570 [hep-ph/9511378] [INSPIRE].

[7] M. Cvetič and P. Langacker, New gauge bosons from string models, Mod. Phys. Lett. A 11 (1996) 1247 [hep-ph/9602424] [INSPIRE].

[8] M. Cvetič, D.A. Demir, J.R. Espinosa, L.L. Everett and P. Langacker, Electroweak breaking and the mu problem in supergravity models with an additional U(1), Phys. Rev. D 56 (1997) 2861 [Erratum ibid. D 58 (1998) 119905] [hep-ph/9703317] [INSPIRE].

[9] D. Ghilencea, L. Ibáñez, N. Irges and F. Quevedo, TeV scale Z' bosons from D-branes, JHEP 08 (2002) 016 [hep-ph/0205083] [INSPIRE].

[10] P. Anastasopoulos, M. Bianchi, E. Dudas and E. Kiritsis, Anomalies, anomalous U(1)'s and generalized Chern-Simons terms, JHEP 11 (2006) 057 [hep-th/0605225] [INSPIRE].

[11] C. Corianò, N. Irges and E. Kiritsis, On the effective theory of low scale orientifold string vacua, Nucl. Phys. B 746 (2006) 77 [hep-ph/0510332] [INSPIRE].

[12] P. Anastasopoulos et al., Minimal anomalous $\mathrm{U}(1)^{\prime}$ extension of the MSSM, Phys. Rev. D 78 (2008) 085014 [arXiv:0804.1156] [INSPIRE].

[13] C. Corianò, N. Irges and S. Morelli, Stückelberg axions and the effective action of anomalous Abelian models. 1. A unitarity analysis of the Higgs-axion mixing, JHEP 07 (2007) 008 [hep-ph/0701010] [INSPIRE].

[14] N. Irges, C. Corianò and S. Morelli, Stückelberg Axions and the Effective Action of Anomalous Abelian Models 2. A SU(3) $C \times \mathrm{SU}(2) W \times \mathrm{U}(1) Y \times \mathrm{U}(1) B$ model and its signature at the LHC, Nucl. Phys. B 789 (2008) 133 [hep-ph/0703127] [INSPIRE].

[15] C. Corianò, M. Guzzi and S. Morelli, Unitarity bounds for gauged axionic interactions and the Green-Schwarz mechanism, Eur. Phys. J. C 55 (2008) 629 [arXiv:0801.2949] [INSPIRE].

[16] J. Kumar, A. Rajaraman and J.D. Wells, Probing the Green-Schwarz mechanism at the Large Hadron Collider, Phys. Rev. D 77 (2008) 066011 [arXiv:0707.3488] [InSPIRE].

[17] I. Antoniadis, A. Boyarsky, S. Espahbodi, O. Ruchayskiy and J.D. Wells, Anomaly driven signatures of new invisible physics at the Large Hadron Collider, Nucl. Phys. B 824 (2010) 296 [arXiv:0901.0639] [INSPIRE].

[18] Y. Mambrini, A clear dark matter gamma ray line generated by the Green-Schwarz mechanism, JCAP 12 (2009) 005 [arXiv:0907.2918] [INSPIRE]. 
[19] G. Shiu, P. Soler and F. Ye, Millicharged dark matter in quantum gravity and string theory, Phys. Rev. Lett. 110 (2013) 241304 [arXiv:1302.5471] [INSPIRE].

[20] M. Cvetič, J. Halverson and H. Piragua, Stringy hidden valleys, JHEP 02 (2013) 005 [arXiv: 1210.5245] [INSPIRE].

[21] B. Körs and P. Nath, A Stückelberg extension of the standard model, Phys. Lett. B 586 (2004) 366 [hep-ph/0402047] [INSPIRE].

[22] B. Körs and P. Nath, A supersymmetric Stückelberg U(1) extension of the MSSM, JHEP 12 (2004) 005 [hep-ph/0406167] [INSPIRE].

[23] D. Feldman, Z. Liu and P. Nath, The Stückelberg $Z^{\prime}$ extension with kinetic mixing and milli-charged dark matter from the hidden sector, Phys. Rev. D 75 (2007) 115001 [hep-ph/0702123] [INSPIRE].

[24] D. Feldman, B. Körs and P. Nath, Extra-weakly interacting dark matter, Phys. Rev. D 75 (2007) 023503 [hep-ph/0610133] [INSPIRE].

[25] E. Dudas, Y. Mambrini, S. Pokorski and A. Romagnoni, (In)visible $Z^{\prime}$ and dark matter, JHEP 08 (2009) 014 [arXiv:0904.1745] [INSPIRE].

[26] E. Dudas, Y. Mambrini, S. Pokorski and A. Romagnoni, Extra U(1) as natural source of a monochromatic gamma ray line, JHEP 10 (2012) 123 [arXiv:1205.1520] [INSPIRE].

[27] C. Jackson, G. Servant, G. Shaughnessy, T.M. Tait and M. Taoso, Higgs in space!, JCAP 04 (2010) 004 [arXiv:0912.0004] [INSPIRE].

[28] C.B. Jackson, G. Servant, G. Shaughnessy, T.M.P. Tait and M. Taoso, Gamma-ray lines and one-loop continuum from s-channel dark matter annihilations, JCAP 07 (2013) 021 [arXiv: 1302.1802] [INSPIRE].

[29] M. Gustafsson, E. Lundstrom, L. Bergstrom and J. Edsjo, Significant gamma lines from inert Higgs dark matter, Phys. Rev. Lett. 99 (2007) 041301 [astro-ph/0703512] [INSPIRE].

[30] T. Bringmann, X. Huang, A. Ibarra, S. Vogl and C. Weniger, Fermi LAT search for internal bremsstrahlung signatures from dark matter annihilation, JCAP 07 (2012) 054 [arXiv: 1203.1312] [INSPIRE].

[31] C. Weniger, A tentative gamma-ray line from dark matter annihilation at the Fermi Large Area Telescope, JCAP 08 (2012) 007 [arXiv: 1204.2797] [INSPIRE].

[32] C. Weniger, M. Su, D.P. Finkbeiner, T. Bringmann and N. Mirabal, Closing in on the Fermi line with a new observation strategy, arXiv:1305.4710 [INSPIRE].

[33] B. de Wit, P. Lauwers and A. Van Proeyen, Lagrangians of $N=2$ supergravity-matter systems, Nucl. Phys. B 255 (1985) 569 [INSPIRE].

[34] I. Antoniadis, E. Kiritsis and T. Tomaras, A D-brane alternative to unification, Phys. Lett. B 486 (2000) 186 [hep-ph/0004214] [INSPIRE].

[35] E. Dudas, A. Falkowski and S. Pokorski, Deconstructed U(1) and supersymmetry breaking, Phys. Lett. B 568 (2003) 281 [hep-th/0303155] [INSPIRE].

[36] L. Andrianopoli, S. Ferrara and M. Lledó, Axion gauge symmetries and generalized Chern-Simons terms in $N=1$ supersymmetric theories, JHEP 04 (2004) 005 [hep-th/0402142] [INSPIRE]. 
[37] E. Dudas, T. Gherghetta and S. Groot Nibbelink, Vector/tensor duality in the five dimensional supersymmetric Green-Schwarz mechanism, Phys. Rev. D 70 (2004) 086012 [hep-th/0404094] [INSPIRE].

[38] J. De Rydt, J. Rosseel, T.T. Schmidt, A. Van Proeyen and M. Zagermann, Symplectic structure of $N=1$ supergravity with anomalies and Chern-Simons terms, Class. Quant. Grav. 24 (2007) 5201 [arXiv:0705.4216] [INSPIRE].

[39] J. De Rydt, T.T. Schmidt, M. Trigiante, A. Van Proeyen and M. Zagermann, Electric/magnetic duality for chiral gauge theories with anomaly cancellation, JHEP 12 (2008) 105 [arXiv:0808.2130] [INSPIRE].

[40] B. Holdom, Two U(1)'s and epsilon charge shifts, Phys. Lett. B 166 (1986) 196 [InSPIRE].

[41] K.R. Dienes, C.F. Kolda and J. March-Russell, Kinetic mixing and the supersymmetric gauge hierarchy, Nucl. Phys. B 492 (1997) 104 [hep-ph/9610479] [INSPIRE].

[42] S. Abel, M. Goodsell, J. Jaeckel, V. Khoze and A. Ringwald, Kinetic mixing of the photon with hidden $\mathrm{U}(1)$ s in string phenomenology, JHEP 07 (2008) 124 [arXiv:0803.1449] [INSPIRE].

[43] M. Pospelov, Secluded U(1) below the weak scale, Phys. Rev. D 80 (2009) 095002 [arXiv: 0811.1030] [INSPIRE].

[44] E.J. Chun, J.-C. Park and S. Scopel, Dark matter and a new gauge boson through kinetic mixing, JHEP 02 (2011) 100 [arXiv:1011.3300] [INSPIRE].

[45] F. del Aguila, G. Coughlan and M. Quirós, Gauge coupling renormalization with several U(1) factors, Nucl. Phys. B 307 (1988) 633 [Erratum ibid. B 312 (1989) 751] [INSPIRE].

[46] F. del Aguila, M. Masip and M. Pérez-Victoria, Physical parameters and renormalization of $\mathrm{U}(1) a \times U(1) b$ models, Nucl. Phys. B 456 (1995) 531 [hep-ph/9507455] [InSPIRE].

[47] G. Bélanger, F. Boudjema, A. Pukhov and A. Semenov, Dark matter direct detection rate in a generic model with MicrOMEGAs 2.2, Comput. Phys. Commun. 180 (2009) 747 [arXiv:0803.2360] [INSPIRE].

[48] M.J. Strassler and K.M. Zurek, Echoes of a hidden valley at hadron colliders, Phys. Lett. B 651 (2007) 374 [hep-ph/0604261] [INSPIRE].

[49] T. Han, Z. Si, K.M. Zurek and M.J. Strassler, Phenomenology of hidden valleys at hadron colliders, JHEP 07 (2008) 008 [arXiv:0712.2041] [INSPIRE].

[50] X. Chu, T. Hambye, T. Scarna and M.H.G. Tytgat, What if dark matter gamma-ray lines come with gluon lines?, Phys. Rev. D 86 (2012) 083521 [arXiv:1206.2279] [INSPIRE].

[51] Planck collaboration, P. Ade et al., Planck 2013 results. XVI. Cosmological parameters, arXiv: 1303.5076 [INSPIRE].

[52] WMAP collaboration, G. Hinshaw et al., Nine-year Wilkinson Microwave Anisotropy Probe (WMAP) observations: cosmological parameter results, Astrophys. J. Suppl. 208 (2013) 19 [arXiv: 1212.5226] [INSPIRE].

[53] G. Bélanger et al., Indirect search for dark matter with MicrOMEGAs2.4, Comput. Phys. Commun. 182 (2011) 842 [arXiv: 1004.1092] [INSPIRE].

[54] G. Bélanger, F. Boudjema, A. Pukhov and A. Semenov, MicrOMEGAs3.1: a program for calculating dark matter observables, arXiv:1305.0237 [INSPIRE]. 
[55] D. Hooper, C. Kelso and F.S. Queiroz, Stringent and robust constraints on the dark matter annihilation cross section from the region of the galactic center, Astropart. Phys. 46 (2013) 55 [arXiv: 1209.3015] [INSPIRE].

[56] LAT collaboration, M. Ackermann et al., Constraints on the galactic halo dark matter from Fermi-LAT diffuse measurements, Astrophys. J. 761 (2012) 91 [arXiv:1205.6474] [INSPIRE].

[57] FERMi-LAT collaboration, A. Abdo et al., Observations of Milky Way dwarf spheroidal galaxies with the Fermi-LAT detector and constraints on dark matter models, Astrophys. J. 712 (2010) 147 [arXiv:1001.4531] [InSPIRE].

[58] Fermi LAT collaboration, A. Drlica-Wagner, Constraints on dark matter and supersymmetry from LAT observations of dwarf galaxies, arXiv:1210.5558 [INSPIRE].

[59] Y.-L.S. Tsai, Q. Yuan and X. Huang, A generic method to constrain the dark matter model parameters from Fermi observations of dwarf spheroids, JCAP 03 (2013) 018 [arXiv: 1212.3990] [INSPIRE].

[60] A. Egorov and E. Pierpaoli, Constraints on dark matter annihilation by radio observations of M31, Phys. Rev. D 88 (2013) 023504 [arXiv: 1304.0517] [INSPIRE].

[61] R. Laha, K.C.Y. Ng, B. Dasgupta and S. Horiuchi, Galactic center radio constraints on gamma-ray lines from dark matter annihilation, Phys. Rev. D 87 (2013), no. 4043516 [arXiv:1208.5488] [INSPIRE].

[62] Y. Mambrini, M.H. Tytgat, G. Zaharijas and B. Zaldivar, Complementarity of Galactic radio and collider data in constraining WIMP dark matter models, JCAP 11 (2012) 038 [arXiv:1206.2352] [INSPIRE].

[63] Y. Bai, P.J. Fox and R. Harnik, The Tevatron at the frontier of dark matter direct detection, JHEP 12 (2010) 048 [arXiv: 1005.3797] [INSPIRE].

[64] P.J. Fox, R. Harnik, J. Kopp and Y. Tsai, LEP shines light on dark matter, Phys. Rev. D 84 (2011) 014028 [arXiv: 1103.0240] [InSPIRE].

[65] H.M. Lee, M. Park and V. Sanz, Interplay between Fermi gamma-ray lines and collider searches, JHEP 03 (2013) 052 [arXiv: 1212.5647] [INSPIRE].

[66] Y. Mambrini and B. Zaldivar, When LEP and Tevatron combined with WMAP and XENON100 shed light on the nature of dark matter, JCAP 10 (2011) 023 [arXiv:1106.4819] [INSPIRE].

[67] ATLAS collaboration, Search for new phenomena in monojet plus missing transverse momentum final states using $10 \mathrm{fb}^{-1}$ of pp collisions at $\sqrt{s}=8 \mathrm{TeV}$ with the ATLAS detector at the LHC, ATLAS-CONF-2012-147 (2012).

[68] CMS collaboration, Search for new physics in monojet events in pp collisions at $\sqrt{s}=8$ TeV, CMS-PAS-EXO-12-048 (2012).

[69] J. Goodman et al., Constraints on light majorana dark matter from colliders, Phys. Lett. B 695 (2011) 185 [arXiv: 1005.1286] [INSPIRE].

[70] J. Goodman et al., Constraints on dark matter from colliders, Phys. Rev. D 82 (2010) 116010 [arXiv: 1008.1783] [INSPIRE].

[71] A. Rajaraman, W. Shepherd, T.M. Tait and A.M. Wijangco, LHC bounds on interactions of dark matter, Phys. Rev. D 84 (2011) 095013 [arXiv:1108.1196] [InSPIRE]. 
[72] A. Belyaev, N.D. Christensen and A. Pukhov, CalcHEP 3.4 for collider physics within and beyond the standard model, Comput. Phys. Commun. 184 (2013) 1729 [arXiv:1207.6082] [INSPIRE].

[73] S. Andreas, C. Niebuhr and A. Ringwald, New limits on hidden photons from past electron beam dumps, Phys. Rev. D 86 (2012) 095019 [arXiv:1209.6083] [INSPIRE].

[74] S. Andreas, M. Goodsell and A. Ringwald, Dark matter and dark forces from a supersymmetric hidden sector, Phys. Rev. D 87 (2013) 025007 [arXiv:1109.2869] [INSPIRE].

[75] N. Fornengo, P. Panci and M. Regis, Long-range forces in direct dark matter searches, Phys. Rev. D 84 (2011) 115002 [arXiv: 1108.4661] [inSPIRE].

[76] Y. Mambrini, The $Z Z^{\prime}$ kinetic mixing in the light of the recent direct and indirect dark matter searches, JCAP 07 (2011) 009 [arXiv:1104.4799] [INSPIRE].

[77] Y. Mambrini, The kinetic dark-mixing in the light of CoGENT and XENON100, JCAP 09 (2010) 022 [arXiv: 1006.3318] [INSPIRE].

[78] M.T. Frandsen, F. Kahlhoefer, A. Preston, S. Sarkar and K. Schmidt-Hoberg, LHC and Tevatron bounds on the dark matter direct detection cross-section for vector mediators, JHEP 07 (2012) 123 [arXiv:1204.3839] [INSPIRE].

[79] M. Goodsell, J. Jaeckel, J. Redondo and A. Ringwald, Naturally light hidden photons in LARGE volume string compactifications, JHEP 11 (2009) 027 [arXiv:0909.0515] [INSPIRE].

[80] S. Abel, M. Goodsell, J. Jaeckel, V. Khoze and A. Ringwald, Kinetic mixing of the photon with hidden $\mathrm{U}(1) s$ in string phenomenology, JHEP 07 (2008) 124 [arXiv:0803.1449] [INSPIRE].

[81] S.N. Gninenko, Constraints on dark photons from $\pi^{0}$ decays, Phys. Rev. D 87 (2013) 035030 [arXiv: 1301.7555] [INSPIRE].

[82] J.H. Davis and C. Boehm, Searching for GeV-scale new gauge bosons in QGP thermal dilepton production, arXiv:1306.3653 [INSPIRE].

[83] L.D. Landau, On the angular momentum of a two-photon system, Dokl. Akad. Nauk Ser. Fiz. 60 (1948) 207.

[84] C.-N. Yang, Selection rules for the dematerialization of a particle into two photons, Phys. Rev. 77 (1950) 242 [INSPIRE].

[85] W.-Y. Keung, I. Low and J. Shu, Landau-Yang theorem and decays of a $Z^{\prime}$ boson into two $Z$ bosons, Phys. Rev. Lett. 101 (2008) 091802 [arXiv:0806.2864] [INSPIRE].

[86] X. Chu, Y. Mambrini, J. Quevillon and B. Zaldivar, Thermal and non-thermal production of dark matter via $Z^{\prime}$-portal(s), arXiv:1306.4677 [INSPIRE]. 